\title{
Facilitating Interstellar Searches for Simple Amino Alcohols with Accurate Rest Frequencies into the Millimeter-wave Regime: Alaninol, Valinol, and Leucinol
}

\author{
Benjamin E. Arenas ${ }^{1,2}$ (10), Mariyam Fatima ${ }^{1,2}$ (10), Cristóbal Pérez ${ }^{1,2}$ (1) , Sophie Fischer ${ }^{1,3}$, Amanda L. Steber ${ }^{1,2,4}$ (1), and \\ Melanie Schnell ${ }^{1,2,4}$ (iD \\ ${ }^{1}$ Deutsches Elektronen-Synchrotron (DESY), Notkestraße 85, D-22607 Hamburg, Germany; amanda.steber@desy.de \\ ${ }^{2}$ Institut für Physikalische Chemie, Christian-Albrechts-Universität zu Kiel, Max-Eyth-Straße 1, D-24118 Kiel, Germany; melanie.schnell@desy.de \\ Received 2020 April 9; revised 2021 February 14; accepted 2021 February 18; published 2021 May 7
}

\begin{abstract}
The detection of larger complex organic molecules, such as molecules consisting of several functional groups or those which show conformational flexibility, in the interstellar medium could lead to insights into the availability of biomolecules in space. We present the rotational spectroscopic study of three amino alcohols: alaninol, valinol, and leucinol. The spectra were recorded over the $2-110 \mathrm{GHz}$ region, which included the utilization of a newly developed instrument operating between $18-26 \mathrm{GHz}$. We report accurately determined line lists, rotational constants, centrifugal distortion constants, and nuclear quadrupole coupling constants for two conformers of alaninol, four conformers of valinol, and three conformers of leucinol, as well as for several singly substituted heavy-atom isotopologues, which also provide structural insights. Further, a number of spectra of vibrationally excited states were assigned, and the associated motions were revealed with anharmonic frequency calculations. Accurate predictions of rotational transitions into the millimeter-wave regime for all species were made, facilitating searches for these complex molecules by observational facilities such as ALMA. Their detection would establish a new family of interstellar molecules.
\end{abstract}

Unified Astronomy Thesaurus concepts: Pre-biotic astrochemistry (2079); High resolution spectroscopy (2096); Molecular spectroscopy (2095); Spectral line lists (2082); Interstellar medium (847); Interstellar molecules (849)

Supporting material: machine-readable tables

\section{Introduction}

Over 200 molecules (Müller et al. 2005; Endres et al. 2016; McGuire 2018) have already been detected in the interstellar medium (ISM), ${ }^{5}$ and many of these molecules contain biologically relevant functional groups that could become incorporated into larger biomolecular motifs. Examples include alcohols and diols (important for their $-\mathrm{OH}$ groups), simple cyano-containing molecules (thought to be important for the incorporation of nitrogen into complex molecules), and even the simple sugar glycolaldehyde (Hollis et al. 2000).

As a consequence of increasingly sensitive observational facilities, larger and more complex molecules are becoming targets of interstellar searches. For example, recently, branched, chiral, and aromatic molecules were identified in space. The amino alcohols are interesting targets for astronomical detections. The family of molecules contains two functional groups - the amino group and the alcohol group - both of which have already been identified in interstellar space. The novel identification of this family of compounds in the ISM is the natural next step in molecular complexity. Molecules that show conformational flexibility, which is another degree of complexity that can be exhibited by larger and branched molecules, are also important targets, and the amino alcohols, particularly the larger molecules, are such examples.

This study aims at providing a complete rotational spectroscopic characterization for these molecules to enable their

\footnotetext{
${ }^{3}$ Current address: Department of Informatics, University of Bergen, Thormøhlensgate 55, NO-5008 Bergen, Norway

${ }_{5}^{4}$ Corresponding author.

5 See the Cologne Database for Molecular Spectroscopy (CDMS), https:// cdms.astro.uni-koeln.de/cdms/portal.
}

detection in interstellar space using the modern, highsensitivity telescopes available. With three, five, and six carbon atoms (or five, seven, and eight heavy atoms), these molecules are in the typical range of the new species that have been detected in recent years. While their potential formation routes, either in the gas phase or on ice grains, might still be an open question, the observation of other molecules containing amino or hydroxyl groups or even both (for example, hydroxylamine) makes a search for these amino alcohols promising.

We also aim at unraveling the conformational flexibility of these molecules. It is important for this attribute to be well explored and that accurate line lists for the energetically relevant conformers exist. The reasons for this are twofold. First, the relative energies of the conformers can be considered, which can focus searches on the lower energy conformers which are more likely to be present, depending on the temperature of the environment. Second, accurate column densities of conformationally flexible molecules can be better achieved when all energetically available conformers are considered. The column density of the molecule is then based on the presence of multiple conformers, rather than on the presence of one variant, for example, as described in Zuckerman et al. (1975) and Pearson et al. (1997) for ethanol.

There have been several previous studies on smaller amino alcohols for the purposes of supporting astronomical detections. For example, the millimeter-wavelength spectroscopy experiments of 2-aminomethanol (Widicus et al. 2003), which determined rotational and centrifugal distortion constants for the ground state and a number of vibrationally excited states, extended previous work on the molecule (Penn \& Curl 1971; Kaushik \& Woods 1982). 2-aminomethanol, also known as glycinol, is the simplest amino alcohol that is related to the 
proteinogenic $\alpha$-amino acid glycine. Several larger amino alcohols have also been examined by rotational spectroscopy, including serinol, which was studied as a model system for the lipid sphingosine (Loru et al. 2016). Another extensive survey along these lines involved a group of molecules, consisting of D-allothreoninol, 2-amino-1,3-propanediol, and 1,3-diamino-2propanol, being studied to analyze the competition between cyclic and chain hydrogen-bonded motifs (Zhang et al. 2016).

In a previous structural analysis of an amino alcohol that is analogous to the proteinogenic $\alpha$-amino acid alanine, Ellingsen et al. (1978) reported a microwave spectroscopy investigation of alaninol with transitions between 18.6 and $39.7 \mathrm{GHz}$. Their experimental analysis revealed the two lowest energy conformers, each with an intramolecular hydrogen bond. They also observed the pure rotational spectra of vibrationally excited states, four for Rotamer I and two for Rotamer II, which can facilitate their radioastronomical detection. The work was followed by a matrix-isolation infrared and Raman spectroscopy study from Fausto et al. (2000), where three further conformations were identified.

Amino alcohols and amino acids are appealing targets for interstellar searches, as important questions in astrochemistry are whether an essential amino acid exists in the gas phase in the ISM and, if so, what are its possible formation pathways. Searches for amino acids in the ISM are not new, with the first reported observation of interstellar glycine in 2003 (Kuan et al. 2003). Despite this observation eventually being disconfirmed (Snyder et al. 2005; Cunningham et al. 2007; Jones et al. 2007), amino acids are still of paramount interest in interstellar (bio) chemistry. The amino alcohols are chemically related to amino acids and essentially represent a reduced state of the corresponding acid; that is, the acid can be produced from the alcohol via an oxidation process. In the gas phase and under radiative conditions, one could speculate that the alcohol species might be more stable than the carboxylic acid counterparts, which are likely to lose $\mathrm{CO}_{2}$.

Several small proteinogenic $\alpha$-amino acids have been characterized by microwave spectroscopy, including alanine, valine, and leucine (Blanco et al. 2004; Lesarri et al. 2004; Cocinero et al. 2007). Each work deduced that the gas-phase structure of the lowest energy conformers of the amino acids contains a bifurcated hydrogen bond between the hydrogen atom of the amino group and the carboxyl oxygen. The portfolio of work also provides the rotational constants required to search for the respective amino acids in the ISM. This has been noteworthy recently because of the detection of glycine, the simplest amino acid, in the coma of the comet 67P/ Churyumov-Gerasimenko (Altwegg et al. 2016).

We present here a study of three potential proteinogenic $\alpha$ amino acid precursors-alaninol (2-amino-1-propanol), valinol (2-amino-3-methyl-1-butanol), and leucinol (2-amino-4-methyl-1pentanol) - with high-resolution, broadband rotational spectroscopy, also known as molecular rotational resonance spectroscopy. Additionally, we link this to our work on isoleucinol (2-amino-3methyl-1-pentanol; Fatima et al. 2020). The rotational spectra of these molecules are investigated across various bands of the $2-110 \mathrm{GHz}$ region to identify and assign vibronic ground-state conformers, singly substituted heavy-atom isotopologues, and vibrationally excited states.

These assignments will facilitate searches for these COMs, which are chemical relatives of amino acids, in the ISM by providing rotational constants and line lists, which are essential for their astronomical detections. Their presence would signify the observation of a new class of molecules not detected in the ISM before, and the successful detection of these amino alcohols could act as a signpost toward regions of the ISM where their amino acid analogs are more likely to be observed.

\section{Experimental Details}

The rotational spectra of alaninol, valinol, and leucinol were recorded with our three chirped-pulse Fourier transform rotational spectrometers. The low-energy conformers were first identified using a supersonic expansion molecular beam on the Hamburg COMPACT spectrometer, between $2-8 \mathrm{GHz}$ (Schmitz et al. 2012; Pérez et al. 2016) and 8-18 GHz (Fatima et al. 2020), and a newly built 18-26 GHz spectrometer (Fatima et al. 2020). The experiments in the millimeter-wave (mmw) regime were carried out on our $W$-band $(75-110 \mathrm{GHz})$ spectrometer (Arenas et al. 2017) at room temperature in a slow flow cell configuration. The spectrometers are summarized here for reference, with individual details for each spectrometer in the following paragraphs. Extensive descriptions of the apparatuses can be found in the corresponding publications.

In short, all three spectrometers are chirped-pulse instruments that use an arbitrary waveform generator (AWG) to produce excitation pulses. These excitation pulses are transmitted into a vacuum chamber, where, when a frequency of the chirped excitation pulse is resonant with a molecular rotational transition, a macroscopic polarization is induced. The resulting free induction decay (FID) of the gaseous sample is collected and fast-Fourier-transformed into the frequency domain. The number of coadded FIDs collected within each frequency range per molecule varied between 0.5 and 5.6 million, and an exact breakdown is given in Table A1. The samples were obtained from Alfa Aesar (L-alaninol, 98\% purity), Biozol (L-valinol, 98\% purity), and Fisher Scientific (L-leucinol, 98\% purity) and were used without further purification. The samples were heated to the following temperatures during the experiments in order to increase their vapor pressures: $80^{\circ} \mathrm{C}$ for alaninol, $30^{\circ} \mathrm{C}$ for valinol, and $125^{\circ} \mathrm{C}$ for leucinol.

In the Hamburg COMPACT spectrometer, the excitation chirps cover the $2-18 \mathrm{GHz}$ range in two separate arrangements. Before transmission into the chamber, the $2-8 \mathrm{GHz}$ excitation pulses are amplified by a $300 \mathrm{~W}$ traveling wave tube amplifier (Schmitz et al. 2012; Pérez et al. 2016). For the $8-18 \mathrm{GHz}$ region, a $50 \mathrm{~W}$ solid-state amplifier is used to amplify the pulses, and to optimize the power per $\mathrm{GHz}$ in this range, the experiment is subdivided into three different frequency regions -8-13, 13-15.5, and 15.5-18 GHz (Fatima et al. 2020). The excitation chirps were $4 \mu \mathrm{s} \quad(2-8 \mathrm{GHz})$ and $2 \mu \mathrm{s}$ long $(8-18 \mathrm{GHz})$, and the FIDs were recorded for $40 \mu \mathrm{s}(2-8 \mathrm{GHz})$ or $20 \mu \mathrm{s}(8-18 \mathrm{GHz})$. The instrument has a resolution of $25 \mathrm{kHz}$ for the $2-8 \mathrm{GHz}$ band and $50 \mathrm{kHz}$ for the $8-18 \mathrm{GHz}$ band. The samples were placed in a heatable reservoir nozzle (Parker Series 9 nozzle), and the molecular vapor was supersonically expanded into the vacuum chamber with 3 bar of neon as the backing gas, which results in a sample rotational temperature of approximately $3 \mathrm{~K}$.

The newly designed $18-26 \mathrm{GHz}$ spectrometer combines the segmented approach and the multi-train method. The segmented approach involves splitting the bandwidth into smaller segments; in this case, the segments are $800 \mathrm{MHz}$ wide, and the spectrum across the whole bandwidth is obtained by stitching 
(a)<smiles>C[C@H](N)[CH]O</smiles>

(b)
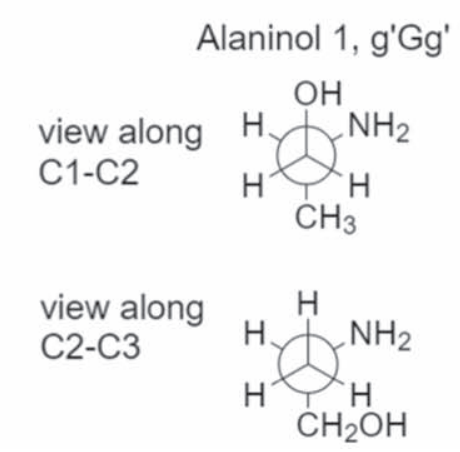

Lewis Structures<smiles>CC(C)(C)C(N)CO</smiles>

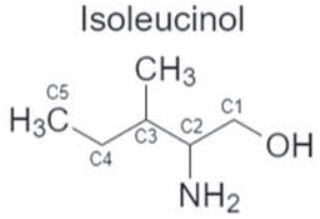

Newman Projections of the Lowest Energy Conformers

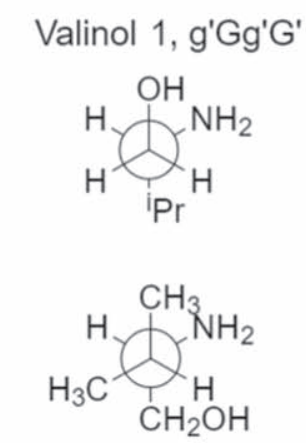

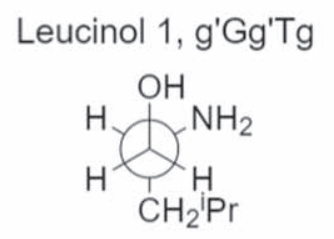

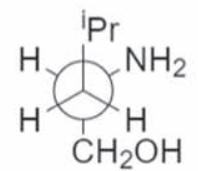

Isoleucinol 1, g'Gg'Tt
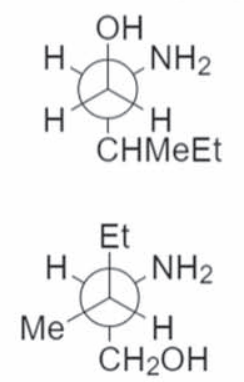

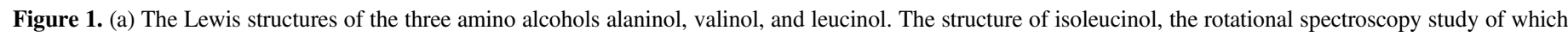

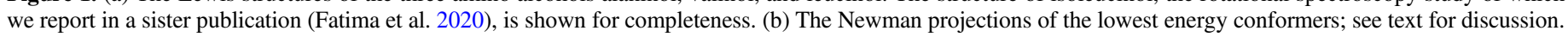

the segments together (Neill et al. 2013). The multi-train method involves utilizing multiple pulse trains per molecular pulse, which increases the effective repetition rate of the experiment (Fatima et al. 2020). The excitation pulses in the 3-7 GHz range, which are $1.5 \mu$ s in duration, are created by an AWG. The pulses are then frequency upconverted to $18-26 \mathrm{GHz}$ with a number of mixing and doubling stages. The FIDs for each segment were collected, downconverted to 400-1200 MHz, and recorded for $10 \mu \mathrm{s}$. Each segment was then corrected to its original frequency by the addition or subtraction of a local oscillator frequency, which was followed by segment concatenation, giving the spectrum across the whole band. The resulting resolution of the spectrometer is approximately $100 \mathrm{kHz}$. Full details of this instrument appear in Fatima et al. (2020). The sample was placed in a similar heatable reservoir nozzle as in the COMPACT. Neon, with a backing pressure of $1 \mathrm{bar}$, was used as the carrier gas.

The commercial $W$-band spectrometer from BrightSpec, Inc., operates in the frequency range $75-110 \mathrm{GHz}$, and it also uses the segmented approach (Neill et al. 2013; Arenas et al. 2017). The AWG produces excitation pulses initially in the range $1.6-2.3 \mathrm{GHz}$, which are then frequency upconverted and multiplied through a series of mixing stages and an active multiplier chain (x6). The instrument's High Dynamic Range mode (Arenas et al. 2017) was used to collect the spectra shown here. Each segment was $30 \mathrm{MHz}$ wide, the pulse duration was $500 \mathrm{~ns}$, and the FID was recorded for $4 \mu \mathrm{s}$. The spectrometer has a resolution of approximately $550 \mathrm{kHz}$. The room-temperature (approximately $300 \mathrm{~K}$ ), high-frequency experiments were performed under slow flow cell conditions, with the sample being pretreated in a freeze-pump-thaw manner to remove any air from the external reservoir. The samples were then heated to be aforementioned temperatures to increase their vapor pressures, vapor was allowed to flow into the chamber without a backing gas, and a pressure of $10 \mu$ bar was maintained in the chamber for all samples.

\section{Theoretical Work}

All three molecules studied here are flexible; rotations around their $\mathrm{C}-\mathrm{C}$ single bonds (see structures in Figure 1(a)) can result in a number of different possible conformations. Conformational searches were conducted for each molecule in the same manner. First, automated semi-empirical conformational searches were done on the SPARTAN program using the Austin Model 1 (AM1) method, the Parameterization Method 3 (PM3), and the Parameterization Method 6 (PM6) (Spartan 14). ${ }^{6}$ After sorting through the structures to remove duplicates, each returned structure was reoptimized with the B3LYP-D3 (BJ) exchange-correlation functional (Becke, three-parameter, Lee-Yang-Parr), including Grimme's empirical dispersion correction with Becke Johnson damping and the def2-TZVP basis set using the Gaussian 09 program package (Frisch et al. 2009). Structures from all three methods that were considered to be energetically accessible to us in our experiments were reoptimized at the B3LYP-D3(BJ)/aug-cc-pVTZ level of theory. The energetic considerations followed those made in Domingos et al. (2016) and focus on the list of potential conformers to those that have energies within $6 \mathrm{~kJ} \mathrm{~mol}^{-1}$ of the lowest energy structure. The number of conformers of each molecule from each method was as follows: alaninol-two conformers from AM1, three conformers from PM3, two conformers from PM6; valinol-four conformers from AM1, four conformers from PM3, three conformers from PM6; leucinol-two conformers from AM1, five conformers from PM3, one conformer from PM6. This resulted in three distinct conformers for alaninol, four distinct conformers for valinol, and five distinct conformers for leucinol, all of which were searched for in the experimental spectra. The automated conformational searches were supplemented with manual dihedral angle scans along the carbon backbones of the molecules. The scans were

\footnotetext{
6 https://www.wavefun.com
} 
Table 1

The Experimental and Theoretical Rotational Constants for the Observed Ground-state Conformers of Alaninol

\begin{tabular}{|c|c|c|c|c|c|c|}
\hline & \multicolumn{3}{|c|}{ Conformer $1, g^{\prime} G g^{\prime}$} & \multicolumn{3}{|c|}{ Conformer $2, g G^{\prime} g$} \\
\hline & This Work & Theory & Ellingsen et al. (1978) & This Work & Theory & Ellingsen et al. (1978) \\
\hline$A / \mathrm{MHz}$ & 7948.48588(88) & 7972.7 & 7948.403(77) & 6461.60804(69) & 6487.9 & $6461.620(32)$ \\
\hline$B / \mathrm{MHz}$ & $3640.57864(28)$ & 3645.7 & $3640.570(11)$ & $4144.43502(37)$ & 4134.6 & $4144.442(30)$ \\
\hline $\mathrm{C} / \mathrm{MHz}$ & 2747.73691(20) & 2749.7 & $2747.727(11)$ & $3336.16546(34)$ & 3328.9 & $3336.163(30)$ \\
\hline$\Delta_{J} / \mathrm{kHz}$ & $0.72345(80)$ & 0.7 & $0.51(18)$ & $1.6343(17)$ & 1.6 & $1.65(69)$ \\
\hline$\Delta_{J K} / \mathrm{kHz}$ & $3.4438(58)$ & 3.4 & $3.84(63)$ & $4.940(10)$ & 5.1 & $6.35(15)$ \\
\hline$\Delta_{K} / \mathrm{kHz}$ & $2.024(87)$ & 2.5 & $1.5(3.2)$ & $-3.701(48)$ & -3.7 & $-7.02(40)$ \\
\hline$\delta_{J} / \mathrm{kHz}$ & $0.17391(29)$ & 0.2 & $0.163(24)$ & $0.24994(53)$ & 0.3 & $0.3420(94)$ \\
\hline$\delta_{K} / \mathrm{kHz}$ & $2.321(13)$ & 2.3 & $3.23(56)$ & $1.115(16)$ & 1.1 & $-0.034(117)$ \\
\hline$\chi_{a a} / \mathrm{MHz}$ & $-3.6734(33)$ & -4.1 & $\ldots$ & $-0.7119(50)$ & -0.8 & $\ldots$ \\
\hline$\chi_{b b}-\chi_{c c} / \mathrm{MHz}$ & $-0.1108(68)$ & -0.18 & $\cdots$ & $4.0368(72)$ & 4.4 & $\cdots$ \\
\hline$\mu_{a}, \mu_{b}, \mu_{c} / \mathrm{D}$ & $\ldots$ & $3.1,0.5,0.4$ & $3.03(1), 0.54(3), 0.57(19)$ & $\ldots$ & $2.5,0.91 .3$ & $2.19(3), 1.46(8), 1.172(3)$ \\
\hline$\kappa$ & -0.66 & -0.66 & -0.66 & -0.48 & -0.49 & -0.48 \\
\hline No. of lines & 258 & $\ldots$ & 39 & 251 & $\ldots$ & 36 \\
\hline$\sigma / \mathrm{kHz}$ & 13.7 & $\cdots$ & 112 & 18.2 & $\cdots$ & 75.8 \\
\hline$J_{\max }, K_{\max }$ & 18,17 & $\ldots$ & $\ldots$ & 15,14 & $\ldots$ & $\ldots$ \\
\hline
\end{tabular}

Note. The experimental values for both conformers are the result of global fits of the data in the regions of 2-18, 18-26, and 75-110 GHz. The theoretical parameters were obtained at the B3LYP-D3(BJ)/aug-cc-pVTZ level of theory. The rotational constants reported by Ellingsen et al. (1978) are included for comparison.

Table 2

The Experimental and Theoretical Rotational Constants for the Observed Ground-state Conformers of Valinol

\begin{tabular}{|c|c|c|c|c|c|c|c|c|}
\hline & \multicolumn{2}{|c|}{ Conformer $1, g^{\prime} G g^{\prime} G^{\prime}$} & \multicolumn{2}{|c|}{ Conformer $2, g^{\prime} G g^{\prime} G$} & \multicolumn{2}{|c|}{ Conformer $3, g^{\prime} G g^{\prime} T$} & \multicolumn{2}{|c|}{ Conformer $4, g G^{\prime} g G^{\prime}$} \\
\hline & This Work & Theory & This Work & Theory & This Work & Theory & This Work & Theory \\
\hline$A / \mathrm{MHz}$ & $4023.53359(37)$ & 4036.8 & $4236.8058(15)$ & 4249.7 & $3629.76830(45)$ & 3651.3 & $3411.43927(47)$ & 3432.0 \\
\hline$B / \mathrm{MHz}$ & $1701.31802(14)$ & 1702.8 & $1546.61067(26)$ & 1549.3 & $1691.76050(54)$ & 1694.5 & 2011.33242(29) & 1998.1 \\
\hline$C / \mathrm{MHz}$ & $1284.21089(12)$ & 1284.2 & $1414.10206(23)$ & 1413.1 & $1470.41499(43)$ & 1466.5 & $1486.51765(23)$ & 1483.0 \\
\hline$\Delta_{J} / \mathrm{kHz}$ & $0.108023(72)$ & 0.1 & $0.22047(15)$ & 0.2 & $0.2656(49)$ & 0.3 & $0.3400(43)$ & 0.4 \\
\hline$\Delta_{J K} / \mathrm{kHz}$ & $0.18277(36)$ & 0.2 & $0.12889(98)$ & 0.1 & $\ldots$ & $\cdots$ & $0.242(24)$ & -0.01 \\
\hline$\Delta_{K} / \mathrm{kHz}$ & $0.724(22)$ & 0.9 & [1.039662972] & 1.0 & $\cdots$ & $\cdots$ & $\ldots$ & $\cdots$ \\
\hline$\delta_{J} / \mathrm{kHz}$ & $0.026377(33)$ & 0.03 & $0.043245(77)$ & 0.04 & $0.0362(29)$ & 0.04 & $0.1216(23)$ & 0.1 \\
\hline$\delta_{K} / \mathrm{kHz}$ & $0.2496(16)$ & 0.3 & $4.825(13)$ & 4.9 & $3.05(15)$ & 1.8 & $\ldots$ & $\ldots$ \\
\hline$\chi_{a a} / \mathrm{MHz}$ & $-4.1801(33)$ & -4.6 & $-3.9918(49)$ & -4.5 & $-4.2541(36)$ & -4.7 & $0.2715(30)$ & 0.5 \\
\hline$\chi_{b b}-\chi_{c c} / \mathrm{MHz}$ & $0.6928(64)$ & 0.6 & $-0.6612(48)$ & -0.6 & $0.7028(76)$ & 0.5 & $4.7808(52)$ & 5.3 \\
\hline$\mu_{a}, \mu_{b}, \mu_{c} / \mathrm{D}$ & $\ldots$ & $3.0,1.2,0.7$ & $\ldots$ & $3.1,0.3,0.7$ & $\ldots$ & $2.8,1.7,0.4$ & $\ldots$ & $1.5,1.7,1.5$ \\
\hline$\kappa$ & -0.70 & -0.70 & -0.91 & -0.90 & -0.79 & -0.79 & -0.45 & -0.47 \\
\hline No. of lines & 569 & $\ldots$ & 172 & $\ldots$ & 184 & $\ldots$ & 258 & $\ldots$ \\
\hline$\sigma / \mathrm{kHz}$ & 20.1 & $\ldots$ & 15.7 & $\ldots$ & 12 & $\ldots$ & 11.8 & $\cdots$ \\
\hline$J_{\max }, K_{\max }$ & 42,42 & $\cdots$ & 36,34 & $\cdots$ & 8,8 & $\cdots$ & 8,8 & $\cdots$ \\
\hline
\end{tabular}

Note. The experimental values for conformers $1, g^{\prime} G g^{\prime} G^{\prime}$, and $2, g^{\prime} G g^{\prime} G$, are the result of global fits of the data in the regions of 2-18, 18-26, and 75-110 GHz. The experimental values for conformers $3, g^{\prime} G g^{\prime} T$, and $4, g G^{\prime} g G^{\prime}$, are the result of fits of the data from 2-26 GHz. The theoretical parameters were obtained at the B3LYP-D3(BJ)/aug-cc-pVTZ level of theory. Values contained within square brackets were kept fixed to the theoretical values.

performed at the B3LYP/6-311G++ level of theory, and each resulting minimum was reoptimized at the B3LYP/aug-cc-pVTZ level of theory. The results from both methods produced the same final list of conformers. The collated list of final conformers was used to aid in spectral assignment. Additionally, for alaninol, we were guided by the conformational landscape reported by Fausto et al. (2000) in their matrix-isolation infrared study. In order to maintain consistency, the naming scheme of the conformers we used was based on the work by Ellingsen et al. (1978) and Fausto et al. (2000), and it is extended here to include the longer carbon chains of leucinol and valinol. The details are included in Appendix B.

Anharmonic frequency calculations, at the B3LYP-D3(BJ)/ aug-cc-pVTZ level of theory, were performed for each assigned conformer in order to obtain theoretical rotational constants for their vibrational states. To aid with the assignments, the calculated rotational constants for the vibrational states were shifted by the difference observed between the experimental and calculated rotational constants for the respective groundstate conformer. The anharmonic frequency calculations also provided theoretical quartic distortion constants to aid in the assignment of the ground-state conformers in the $W$-band data sets.

\section{Analysis}

The amino alcohols studied here are all asymmetric tops, where $A \neq B \neq C$. The theoretical and experimental values for Ray's asymmetry parameter, $\kappa$, for each conformer are given in 
Table 3

The Experimental and Theoretical Rotational Constants for the Observed Ground-state Conformers of Leucinol

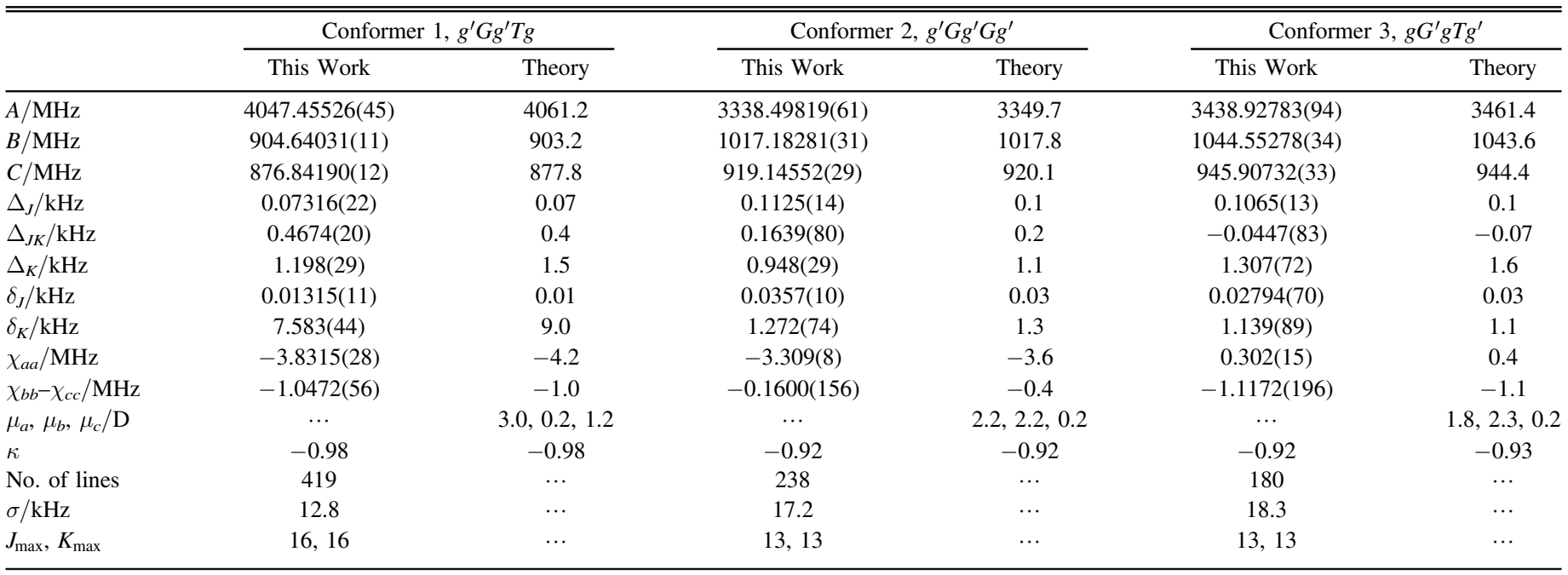

Note. The experimental values for all three conformers are the result of fits of the data from 2-26 GHz. The theoretical parameters were obtained at the B3LYP-D3 (BJ)/aug-cc-pVTZ level of theory.

Tables 1-3, where

$$
\kappa=\frac{2 B-A-C}{A-C} .
$$

Generally, the values of $\kappa$ range from -1 to 1 , where these two limits are indicative of symmetric prolate and symmetric oblate tops, respectively. All of the conformers are asymmetric, near-prolate tops, with the theoretical $\kappa$ values ranging from -0.99 to -0.48 . They were thus fit to a Watson's $A$-reduction Hamiltonian in the $I^{r}$ representation. Spectral analysis was carried out using the PGOPHER program (Western 2017) and the AABS program package (Kisiel et al. 2005; Kisiel 2015). The final fits were obtained from Pickett's suite of programs (SPFIT/SPCAT) (Pickett 1991).

The low-frequency data sets, up to $26 \mathrm{GHz}$, exhibited hyperfine splitting due to the nuclear electric quadrupole moment of the nitrogen nucleus. This hyperfine splitting was only relevant for these data sets, and not for the $W$-band spectra, as the splitting collapses for transitions with a high rotational quantum number, $J$. In order to obtain a fit encompassing all of the measured transitions, a global fit was performed whereby the rotational constants and the quartic centrifugal distortion constants were fit to all assigned lines, and the nitrogen nuclear quadrupole coupling constants were fit only to those lines that exhibited the hyperfine splitting. That is, any transition exhibiting hyperfine splitting was labeled with the relevant $F$-state labels, and those that did not exhibit the splitting were assigned to a pure rotational Hamiltonian. Further details on this can be found in Giuliano et al. (2019), where the same procedure in the analysis of the rotational spectrum of imidazole was used.

The substitution structures of some of the amino alcohols were determinable because singly substituted isotopologues were observed in natural abundance. This analysis was performed using the KRA program package, and further structural analysis, such as the determination of dihedral angles, was performed with the EVAL program package (Kisiel et al. 2005; Kisiel 2015).

\section{Results and Discussions}

The spectroscopic parameters for the observed ground-state conformers of alaninol $\left(g^{\prime} G g^{\prime}\right.$ and $\left.g G^{\prime} g\right)$, valinol $\left(g^{\prime} G g^{\prime} G^{\prime}\right.$, $g^{\prime} G g^{\prime} G, \quad g^{\prime} G g^{\prime} T$, and $\left.g G^{\prime} g G^{\prime}\right)$, and leucinol $\left(g^{\prime} G g^{\prime} T g\right.$, $g^{\prime} G g^{\prime} G g^{\prime}$, and $\left.g G^{\prime} g T g^{\prime}\right)$ are presented in Tables 1-3, respectively. In these tables, the experimentally determined constants are listed alongside those obtained from theory (as described above). The theoretical rotational constants agree with the experimental rotational constants within a $0.4 \%$ difference for alaninol, a $0.6 \%$ difference for valinol, and a $0.7 \%$ difference for leucinol. A comparison to the previously published rotational constants of alaninol (Ellingsen et al. 1978) is also presented in Table 1. The improvement of the microwave rms $(\sigma)$ between the current analysis of alaninol and that performed by Ellingsen et al. (1978) highlights the experimental frequency accuracy available from modern high-resolution rotational spectroscopy. The fits for alaninol 1, $g^{\prime} G g^{\prime}$, alaninol 2, $g G^{\prime} g$, valinol $1, g^{\prime} G g^{\prime} G^{\prime}$, and valinol 2, $g^{\prime} G g^{\prime} G$, are the result of combining data from the ranges 2-26 and $75-110 \mathrm{GHz}$. Valinol $3, g^{\prime} G g^{\prime} T$, and valinol $4, g G^{\prime} g G^{\prime}$, were not assigned in the $W$-band spectrum, likely due to a combination of the low signal-to-noise of these higher energy conformers and difficulties in pinpointing transitions in the confusion-limited spectrum.

Despite extensive efforts, including performing frequency calculations at various levels of theories and basis sets to obtain theoretical rotational and distortion constants, neither the vibronic ground states nor vibrationally excited states of leucinol could be assigned in the room-temperature $W$-band spectrum. Extrapolation of the rotational constants determined from the low-frequency data $(2-26 \mathrm{GHz})$ into the $75-110 \mathrm{GHz}$ region predicts 19 R-branch transition sets $(J=43$ to $J=61)$. But there are no identifiable transition features or patterns in the high-frequency spectrum, indicating that the measured species in the $W$-band experiment was not leucinol, but perhaps a degradation product.

For all systems, the spectra recorded with the COMPACT and the $18-26 \mathrm{GHz}$ spectrometers showed hyperfine splitting, caused by the interaction of the ${ }^{14} \mathrm{~N} I=1$ spin with the electric 
(a)

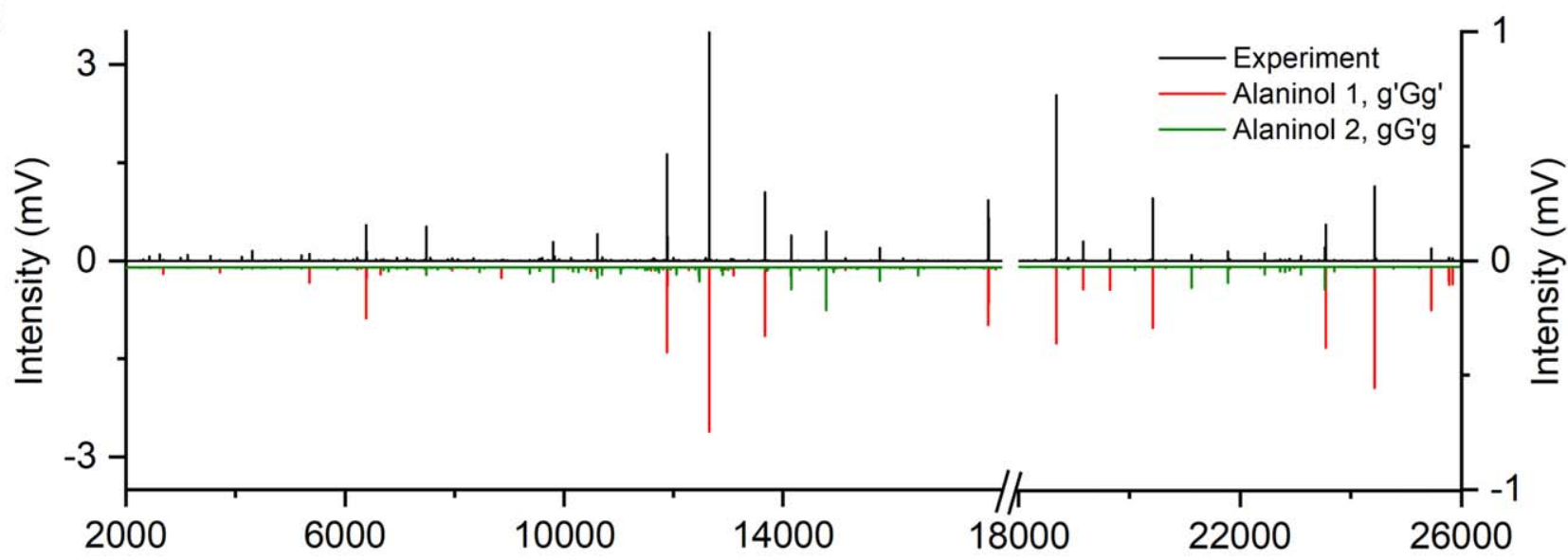

(b)

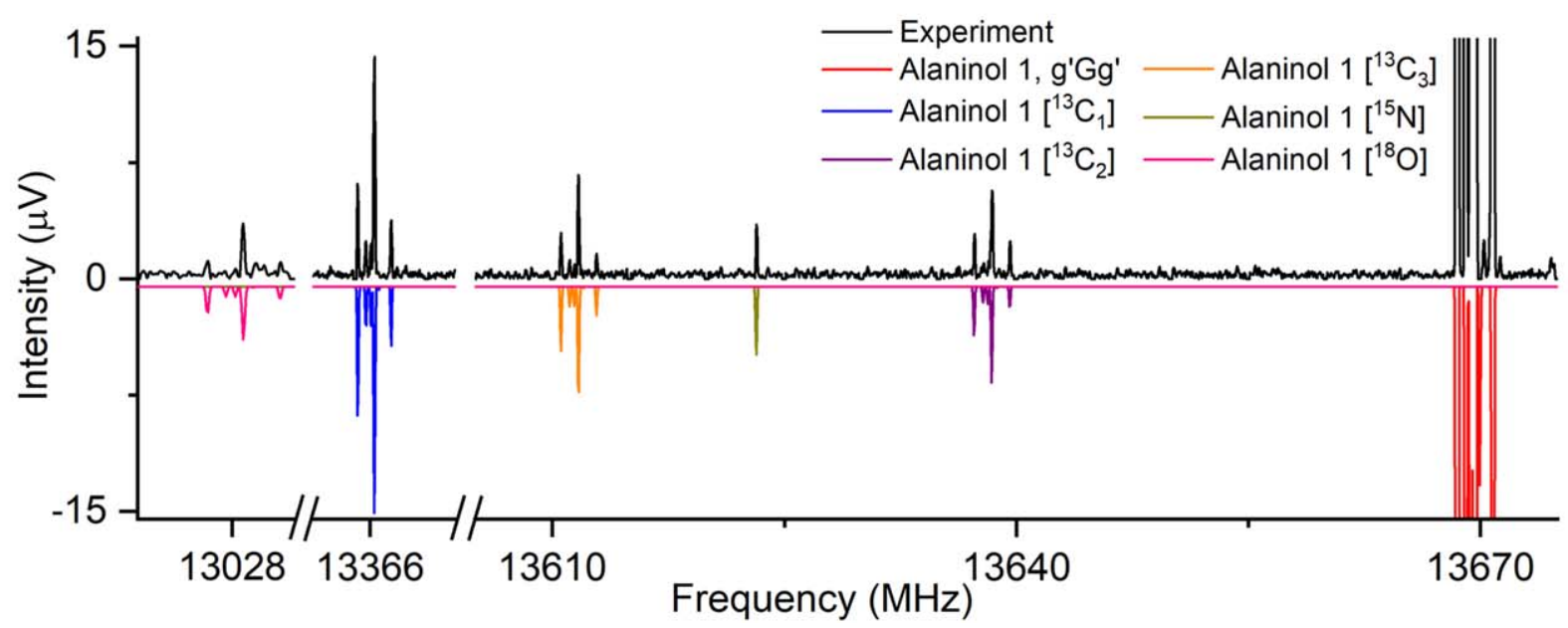

Figure 2. (a) The low-frequency (2-18 and $18-26 \mathrm{GHz})$ experimental data sets of the rotational spectrum of alaninol. The numbers of coadded FIDs, in millions, are $1.9,1.1,1.0,1.4$, and 2.6 in the $2-8,8-13,13-15.5,15.5-18$, and $18-26 \mathrm{GHz}$ regions, respectively. Note the different intensity axes for the two different frequency ranges. (b) Zoom-ins to illustrate the assignments of the observed isotopologues for alaninol $1, g^{\prime} G^{\prime}$, in the $13-15.5 \mathrm{GHz}$ region. In both, the black trace is the experimental spectrum, and the colored traces are simulated spectra based on the fitted rotational constants at $3 \mathrm{~K}$, which was the rotational temperature of the supersonic expansion.

field gradient. This is shown clearly in Figure 2, where a section of the rotational spectrum of alaninol is plotted against the simulated spectrum of the fitted rotational constants for the lowest energy conformer, $g^{\prime} G g^{\prime}$. The hyperfine splitting generally spans $2-3 \mathrm{MHz}$ for alaninol $1, g^{\prime} G g^{\prime}$, and this holds true for the other amino alcohol conformers. Analysis of this effect has allowed us to determine the nitrogen nuclear quadrupole coupling constants $\chi_{a a}$ and $\chi_{b b}-\chi_{c c}$ for all ground-state conformers and a number of isotopologues. Comparison of these experimental constants to those computed from theory provides a further confirmation of the structure designated to each assignment. These constants are of particular importance for interstellar searches, as the observation of nuclear hyperfine splitting can act as a further validation mechanism for interstellar detections (Loomis et al. 2013; McGuire et al. 2018).

In addition to the low-energy conformers listed above, we also observed the rotational spectra of a number of isotopologues in natural abundance in the $2-26 \mathrm{GHz}$ data sets. The application of Kraitchman's equations (Kraitchman 1953) allowed us to experimentally determine the position of each isotopically substituted atom with respect to the molecule's center of mass. A comparison of the experimentally determined substitution structures in the gas phase and the quantumchemically optimized structures is presented in Figure 3 for those that were observed. The partially transparent molecular structure is the optimized structure at the B3LYP-D3(BJ)/augcc-pVTZ level of theory, and the colored spheres are the experimentally determined atom positions. As shown, the theoretical structures match the experimental structures. Isotopologues were observed for the following conformers: ${ }^{13} \mathrm{C},{ }^{15} \mathrm{~N}$, and ${ }^{18} \mathrm{O}$ for alaninol $1\left(g^{\prime} G g^{\prime}\right)$ and valinol 1 $\left(g^{\prime} G g^{\prime} G^{\prime}\right) ;{ }^{13} \mathrm{C}$ and ${ }^{15} \mathrm{~N}$ for alaninol $2\left(g G^{\prime} g\right)$, valinol 2 $\left(g^{\prime} G g^{\prime} G\right)$, and leucinol $1\left(g^{\prime} G g^{\prime} T g\right)$. A full account of the rotational constants for the isotopologues observed is reported in Tables C1-C5. The analysis of the isotopologues allowed us to unambiguously determine the experimental backbones of the alaninol conformers, the two lowest energy conformers of valinol, and the lowest energy conformer of leucinol. Aside from the structural analysis that can be developed from this information, which follows below, this information can be useful to astronomers in the future for searches for these species in the ISM. Searches of this nature would rely on any parent-species conformer being detected in sufficient abundance, and the likeliness of detection would be increased with the continuously improving sensitivities of observational 

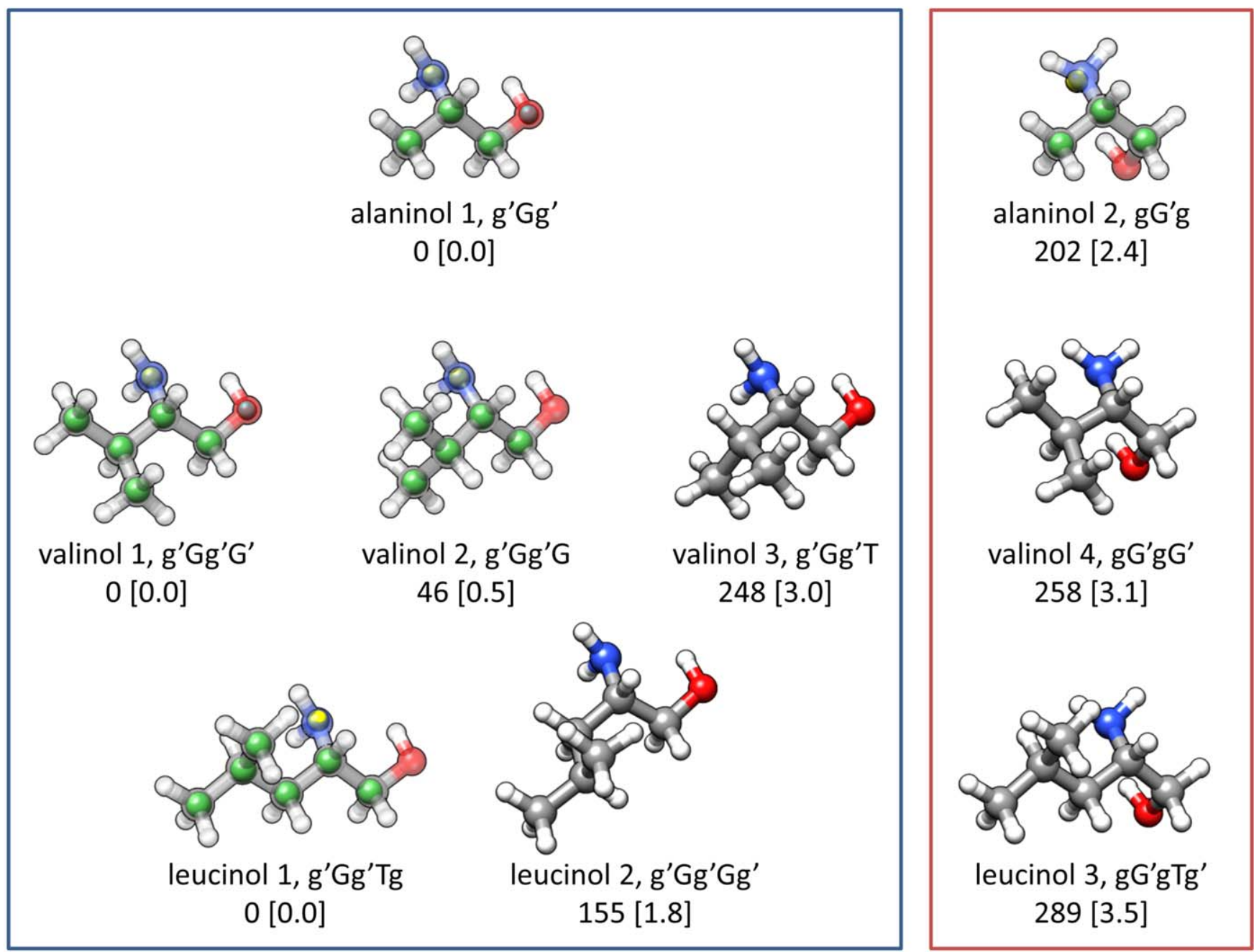

Figure 3. The structures of alaninol, valinol, and leucinol observed in our experiments. The structures shown were optimized at the B3LYP-D3(BJ)/aug-cc-pVTZ level of theory. The zero-point-energy-corrected energy values, in the format $\mathrm{cm}^{-1}\left[\mathrm{~kJ} \mathrm{~mol}^{-1}\right]$, are noted relative to the lowest energy conformer of that particular molecule. Where applicable, a comparison of the experimentally determined atom positions and the atom positions from the structure optimization calculations are shown - the theoretical structures are in faded colors, and the solid spheres are the determined positions for carbon (green), nitrogen (yellow), and oxygen (cyan). The conformers are further subdivided into the two hydrogen-bond motifs named via the aBc dihedral angles-those within the blue box conform to the $g^{\prime} G g^{\prime}$ dihedral angle template, those within the red box conform to the $g G^{\prime} g$ dihedral angle template (see Appendix B for further details).

facilities. Heavy-atom isotopologue detections, despite currently being relatively rare in comparison to detections of mainisotopologue species, could in the future provide information on isotopic fractionation and reveal reaction pathways in specific regions (Furuya et al. 2011).

As can be seen in Figure 3, all of the lowest energy conformers of each amino alcohol have the same backbone in terms of the relative positions of the amino and the alcohol groups in the molecule. That is, they follow the structure of conformer 1 of alaninol, $g^{\prime} G g^{\prime}$, where the conformer is dominated by the hydrogen-bond stabilization between the $-\mathrm{NH}_{2}$ and the $-\mathrm{OH}$ groups. Following the depiction in Figure 1(b), viewing the molecules' stabilized configuration along the $\mathrm{C} 1-\mathrm{C} 2$ bond clearly shows that the relatively large $\mathrm{X}$ substituent is anti to the $-\mathrm{OH}$ group $(-\mathrm{X}=-\mathrm{Me}$ for alaninol, $-{ }^{i} \mathrm{Pr}$ for valinol, $-\mathrm{CH}_{2}^{\mathrm{i}} \mathrm{Pr}$ for leucinol, and $-\mathrm{CHMeEt}$ for isoleucinol). This was first discussed for alaninol by Ellingsen et al. (1978). If the molecule is viewed along the C2-C3 bond, the anti configuration for the $-\mathrm{CH}_{2} \mathrm{OH}$ on $\mathrm{C} 2$ and the bulkiest substituent on $\mathrm{C} 3$ can also be noted. Where there is more than one nonhydrogen substituent on the $\mathrm{C} 3$ position, the second is positioned anti to the $-\mathrm{NH}_{2}$ group. This is seen most clearly in isoleucinol, which was recently published and is included in Figure 1(b) for completeness, where the anti pairs along the $\mathrm{C} 2-\mathrm{C} 3$ bond are $-\mathrm{CH}_{2} \mathrm{OH} /-\mathrm{Et}$ and $-\mathrm{NH}_{2} /-\mathrm{Me}$. The differences between the molecules arise from the various additions to the carbon chain at the $\mathrm{C} 3$ position. One would expect that as more extended carbon groups are added to the $\mathrm{C} 3$ position, the number of low-energy conformers increases due to the higher number of $\mathrm{C}-\mathrm{C}$ single bonds around which bond rotations can occur. However, steric effects do play a role, and this can be seen by the fact that a number of "expected" conformers are not observed; for example, the hypothetical structure of leucinol 1, $g^{\prime} G g^{\prime} T g$, in which the $\mathrm{C} 1-\mathrm{C} 2-\mathrm{C} 3-\mathrm{C} 4$ dihedral angle is around $-60^{\circ}$, is not observed because of steric hindrance. Indeed, this hypothetical structure does not appear as a minimum in our conformational search, as it would likely interconvert to either leucinol $1, g^{\prime} G g^{\prime} T g$, or leucinol 2, $g^{\prime} G g^{\prime} G g^{\prime}$. Lesarri et al. (2004) have already noted steric effects as an important consideration in explaining "missing" conformers. The structures of the amino alcohols follow chemical intuition when considering the anti configurations of the bulky substituents, 
(a)

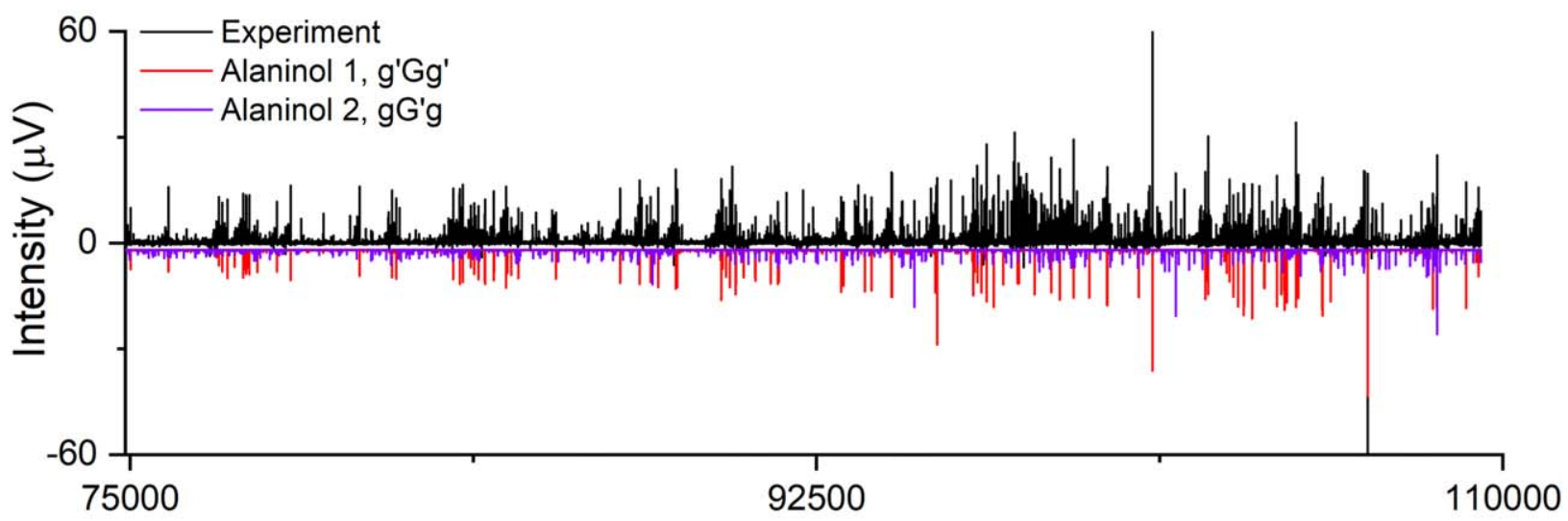

(b)

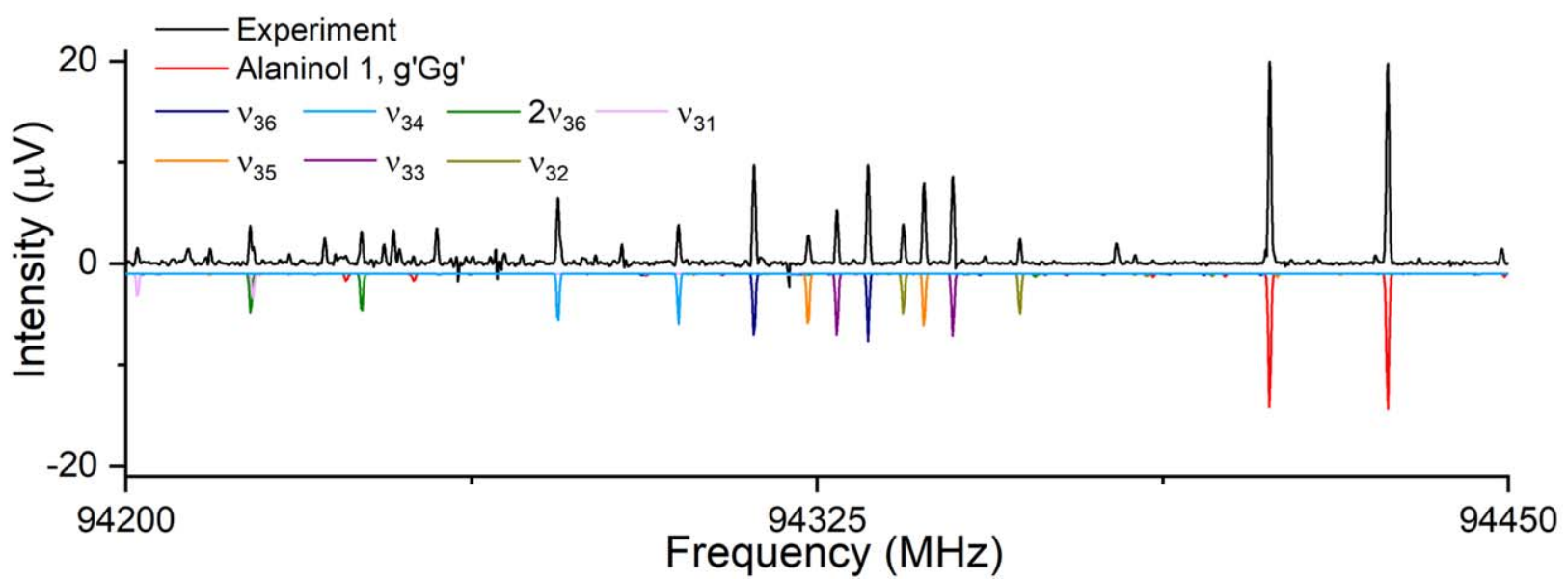

Figure 4. (a) High-frequency rotational spectrum of alaninol. The black trace is the experimental spectrum of 500,000 coadded FIDs, and the colored traces are the simulated spectra based on the fitted rotational constants at $300 \mathrm{~K}$, which was approximately the temperature of the flow cell. (b) Zoom-in illustrating the assignment of alaninol $1, g^{\prime} G g^{\prime}$, and seven fundamental vibrational states of that conformer.

but as already mentioned, steric effects should be considered as an important factor in determining if a structure will be observable in a supersonic expansion.

The studies on the rotational spectra of the relevant amino acids (Blanco et al. 2004; Lesarri et al. 2004; Cocinero et al. 2007) allow us to compare the structural motifs with the amino alcohol structures observed here. The lowest energy structures of all the observed amino acids in the gas phase (Conformer I in the relevant publications) contain a bifurcated hydrogen bond between the amino group and the oxygen of the carbonyl group. With the lack of a carbonyl group in the amino alcohol analogs, it is not surprising that the lowest energy conformers of the amino alcohols are not structurally similar to those of the amino acids. However, in all cases, the lowest energy amino alcohol conformer resembles the motif of the next energetically favorable amino acid conformer observed; that is, alaninol $1, g^{\prime} G g^{\prime}$, resembles alanine IIa, valinol $1, g^{\prime} G g^{\prime} G^{\prime}$, resembles valine IIa, and leucinol 1, $g^{\prime} G g^{\prime} T g$, resembles leucine $\mathrm{IIb}_{1}$. In the same vein, we could show that the lowest energy conformer of isoleucinol, $g^{\prime} G g^{\prime} T t$, is structurally similar to isoleucine $\mathrm{IIa}_{1}$ (Fatima et al. 2020). The major differences between the similar structures can be explained by the constitutional difference regarding the carbonyl group. The amino acids contain the carbonyl group, which itself adopts a planar structure due to the $s p^{2}$-hybridized carbon. In the study on alanine, Blanco et al. (2004) state that "the amino acid backbone is nonplanar," and this nonplanarity is further emphasized in alaninol, wherein the $s p^{2}$-hybridized carbon is now an $s p^{3}$-hybridized carbon. This results in a tetrahedral arrangement around the $\mathrm{C} 1$ atom, and, thus, a nonplanar arrangement of the backbone. The hydrogen bond between the amino group and the alcohol group $(-\mathrm{N} \cdots \mathrm{H}-)$ increases from $1.96(1) \AA$ in alanine IIa (Blanco et al. 2004) to $2.23 \AA$ in alaninol $1, g^{\prime} G g^{\prime}$ (from our structure optimization calculation), and the $\mathrm{N}-\mathrm{C} 2-\mathrm{C} 1-\mathrm{O}$ dihedral angle, the dihedral angle effectively defining the backbone planarity, increases from $-11.5(8)^{\circ}$ in alanine Ila (Blanco et al. 2004) to $55.7(9)^{\circ}$ in alaninol $1, g^{\prime} G g^{\prime}$. The dihedral angle in alaninol is the same as that reported previously, 54(2) ${ }^{\circ}$ (Ellingsen et al. 1978), and it is in keeping with the dihedral angle from the theoretical structure at the B3LYP-D3(BJ)/aug-cc-pVTZ level of theory, which is $53.9^{\circ}$.

There is not as detailed an account of structural parameters in the studies of valine and leucine. This means that valinol and leucinol cannot be directly compared to their amino acid analogs in the same manner as alaninol can be compared to alanine. The experimental $\mathrm{N}-\mathrm{C} 2-\mathrm{C} 1-\mathrm{O}$ dihedral angle for valinol $1, g^{\prime} G g^{\prime} G^{\prime}$, is $50.5(7)^{\circ}$. This compares well with the dihedral angle in the optimized theoretical structure, which is $50.2^{\circ}$. Our study on isoleucinol revealed the same angle to be $50.9(8)^{\circ}$ in isoleucinol $1, g^{\prime} G g^{\prime} T t$, for which the theoretical value for the dihedral angle is $50.4^{\circ}$ (Fatima et al. 2020). The full experimental substitution structure of leucinol $1, g^{\prime} G g^{\prime} T g$, is not currently available, as the ${ }^{18} \mathrm{O}$ isotopologue was not observed in natural abundance. We 
Table 4

The Experimental and Theoretical Rotational Constants for the Observed Vibrational States of the Lowest Energy Conformer of Alaninol $1, g^{\prime} G g^{\prime}$

\begin{tabular}{|c|c|c|c|c|c|c|c|}
\hline & $\nu_{36}$ & $\nu_{35}$ & $\nu_{34}$ & $\nu_{33}$ & $2 \nu_{36}$ & $\nu_{32}$ & $\nu_{31}$ \\
\hline$E^{h} / \mathrm{cm}^{-1}\left[\mathrm{~kJ} \mathrm{~mol}^{-1}\right]$ & $146.3[1.8]$ & 228.7 [2.7] & $257.4[3.1]$ & $266.5[3.2]$ & $292.7[3.5]$ & $365.2[4.4]$ & $451.4[5.4]$ \\
\hline$E^{a} / \mathrm{cm}^{-1}\left[\mathrm{~kJ} \mathrm{~mol}^{-1}\right]$ & $151.8[1.8]$ & $219.3[2.6]$ & $251.8[3.0]$ & $262.8[3.1]$ & $306.1[3.7]$ & $363.9[4.4]$ & $457.4[5.5]$ \\
\hline$A_{\text {theory }} / \mathrm{MHz}$ & 7907.5 & 7930.8 & 7957.6 & 7965.8 & 7866.4 & 7952.8 & 7940.7 \\
\hline$B_{\text {theory }} / \mathrm{MHz}$ & 3637.5 & 3637.8 & 3638.9 & 3638.7 & 3634.4 & 3640.8 & 3638.0 \\
\hline$C_{\text {theory }} / \mathrm{MHz}$ & 2746.2 & 2745.4 & 2742.6 & 2744.2 & 2744.6 & 2745.6 & 2747.0 \\
\hline$A_{\mathrm{exp}} / \mathrm{MHz}$ & $7907.215(19)$ & $7930.202(22)$ & $7971.968(21)$ & $7948.078(15)$ & $7873.565(20)$ & $7946.558(21)$ & $7922.836(21)$ \\
\hline$B_{\exp } / \mathrm{MHz}$ & $3636.4881(14)$ & $3638.1177(18)$ & $3638.3710(15)$ & $3641.2328(12)$ & $3632.5727(16)$ & $3640.4956(15)$ & 3634.6192(19) \\
\hline$C_{\exp } / \mathrm{MHz}$ & $2745.8447(13)$ & $2745.4712(17)$ & $2742.8480(13)$ & $2744.8387(10)$ & $2743.8372(13)$ & $2745.4025(13)$ & $2741.7037(13)$ \\
\hline$\Delta_{J} / \mathrm{kHz}$ & $0.7308(17)$ & $0.7200(23)$ & $0.7151(19)$ & $0.7222(17)$ & $0.7393(22)$ & $0.7202(22)$ & $0.7209(28)$ \\
\hline$\Delta_{J K} / \mathrm{kHz}$ & $3.4823(82)$ & $3.581(12)$ & $3.3912(93)$ & $3.5434(83)$ & $3.500(10)$ & $3.442(13)$ & $3.497(14)$ \\
\hline$\Delta_{K} / \mathrm{kHz}$ & $1.77(51)$ & $2.06(67)$ & $2.49(58)$ & $2.16(44)$ & $2.06(62)$ & $2.60(65)$ & $2.27(69)$ \\
\hline$\delta_{J} / \mathrm{kHz}$ & $0.1766(11)$ & $0.1660(16)$ & $0.1765(12)$ & $0.1738(12)$ & $0.1769(14)$ & $0.1737(14)$ & $0.1788(19)$ \\
\hline$\delta_{K} / \mathrm{kHz}$ & $2.239(31)$ & $2.191(39)$ & $2.539(30)$ & $2.373(26)$ & $2.335(44)$ & $2.294(46)$ & $2.563(39)$ \\
\hline No. of lines & 78 & 53 & 69 & 68 & 65 & 55 & 51 \\
\hline$\sigma / \mathrm{kHz}$ & 24.3 & 22.5 & 25.5 & 18.8 & 23.4 & 22.9 & 22.5 \\
\hline
\end{tabular}

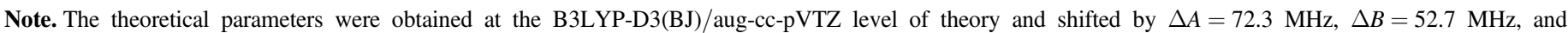

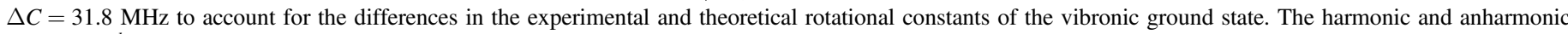
energies ( $E^{h}$ and $E^{a}$ respectively) of the vibrational states tabulated are relative to the energy of the alaninol $1, g^{\prime} G g^{\prime}$, conformer.

expect, however, that the theoretical value $\left(53.7^{\circ}\right)$ will be an adequate estimation because of the agreement between experimental and theoretical values for the other amino alcohols in the series and the good agreement between experimental and theoretical rotational constants. All these values are in keeping with the dihedral angle in alaninol $1, g^{\prime} G g^{\prime}$, and they show a $3.8 \%$ average deviation from the mean value. We speculate that these small deviations in the dihedral angles originate from the different groups on the $\mathrm{C} 3$ atoms of the molecules. In valinol and isoleucinol, the groups on the $\mathrm{C} 3$ atom could exhibit steric hindrance with the groups on the $\mathrm{C} 2$ atom, which would make the $\mathrm{N}-\mathrm{C} 2-\mathrm{C} 1-\mathrm{O}$ dihedral angle deviate more from $60^{\circ}$. This effect is less pronounced for alaninol and leucinol, which both have fewer bulky groups on the $\mathrm{C} 3$ atom. This can be seen in the Newman projections in Figure 1(b) and follows from the earlier discussion about the projections. The dihedral angle $\mathrm{N}-\mathrm{C} 2-\mathrm{C} 1-$ $\mathrm{O}$ effectively defines the carbon backbone planarity in the amino alcohols studied, and the values reported here indicate nonplanarity. Where data are available, an increase in the degree of nonplanarity is seen when compared to the analogous amino acid.

The spectroscopic data for the vibrational states were accessible because the $W$-band experiments were performed at room temperature. As stated previously, they could prove important if the ground-state conformers are detected in warm regions of the ISM. The assignments of the vibrational states of alaninol conformer $1, g^{\prime} G g^{\prime}$, are shown in the zoom-ins in Figure 4. A short description of the fundamental modes follows: $\nu_{36}$ is the heavy-atom torsion, $\nu_{35}$ is the rotation of the terminal methyl group, $\nu_{34}$ is a scissor motion of the carbon chain, $\nu_{33}$ is a motion in the $-\mathrm{NH}_{2}$ group, $\nu_{32}$ is the $-\mathrm{CH}_{3} /-\mathrm{NH}_{2}$ scissor, and $\nu_{31}$ is a wagging motion mainly affecting the hydrogens on terminal atoms. Figure D1 shows these motions alongside their energies with anharmonic corrections at the B3LYP-D3(BJ)/aug-cc-pVTZ level of theory. The rotational constants derived for these, as well as the combination band $2 \nu_{36}$, are detailed in Table 4 . Short descriptions of the motions involved for the vibrations of valinol can also be found in Appendix D. In total, the number of assigned vibrational states for each molecule, by conformer, is 7 for alaninol $1, g^{\prime} G^{\prime} g ; 1$ for alaninol $2 ; g G^{\prime} g ; 3$ for valinol 1 , $g^{\prime} G g^{\prime} G^{\prime}$. The experimental rotational constants for the observed vibrational states of alaninol $2, g G^{\prime} g$, and valinol 1 , $g^{\prime} G g^{\prime} G^{\prime}$, can be found in Tables D1 and D2. The shifted theoretical rotational constants (see Section 3 for details) are included in the tables, which were gleaned from the anharmonic frequency calculations we performed. A direct application of this work is that detected vibrational states could be used as temperature probes for regions where the groundstate conformers are detected, for example, in hot core regions. Further, the elimination of lines from vibrationally excited states (and isotopologues), which can occupy many channels in astronomical data sets, is an important step in making complex observational spectra more useful by removing lines from already-known molecules.

Searches for the assigned species in this work in observational data sets require not only accurate rest frequencies but also information on the relative intensities of the rotational transitions. Partition functions can be used to calculate the absorption coefficients and hence transition intensities. This information can be further used to calculate column densities of detected species (Mangum \& Shirley 2015). The rotational partition functions computed across the standard temperatures implemented in the JPL database (Pickett et al. 1998) are reported in Table 5 for the lowest energy conformer of alaninol $1, g^{\prime} G g^{\prime}$. Two sets of rotational partition functions are tabulated: one for spin degeneracy equal to $1(2 I+1=1$; the case where hyperfine splitting is not considered) and one for spin degeneracy equal to $3(2 I+1=3$; the case where hyperfine splitting is considered ). A consequence of varying temperature is the change in the intensity distribution of the observed rotational transitions. This can be seen in Figure 5, which shows the simulated spectra for alaninol $1, g^{\prime} G g^{\prime}$, across a number of temperatures. This plot was made using the rotational partition functions determined for the case where the spin degeneracy is equal to 1 . The general distribution of transitions is the same for the case where the spin degeneracy is equal to 3 . The rotational partition functions for the other assigned species-the ground-state conformers alaninol 


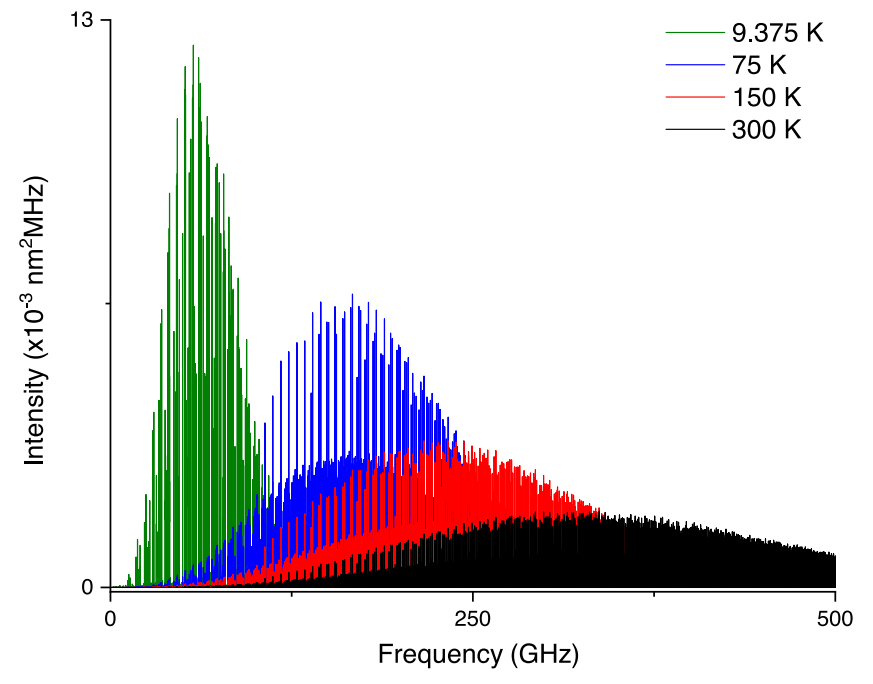

Figure 5. The distributions of rotational transitions of alaninol $1, g^{\prime} G g^{\prime}$, across four of the standard temperatures implemented in the JPL database: $9.375 \mathrm{~K}$, $75 \mathrm{~K}, 150 \mathrm{~K}$, and $300 \mathrm{~K}$ (Pickett et al. 1998). The maximum frequency simulated here is $500 \mathrm{GHz}$, and the plot was made for the case where $2 I+1=1$. Note that the intensity scale for the prediction at $9.375 \mathrm{~K}$ has been halved for a clearer comparison to the other predictions.

2, $g G^{\prime} g$, valinol $1, g^{\prime} G g^{\prime} \mathrm{G}^{\prime}$, valinol $2, g^{\prime} G g^{\prime} G$, valinol 3, $g^{\prime} G g^{\prime} T$, valinol 4, $g G^{\prime} g G^{\prime}$, leucinol $1, g^{\prime} G g^{\prime} T g$, leucinol $2, g^{\prime} G g^{\prime} G g^{\prime}$, leucinol 3, $g G^{\prime} g T g^{\prime}$, the heavy-atom isotopologues, and the vibrationally excited states_can be found in Tables E1-E2. The rotational partition functions were all calculated using the SPCAT program (Pickett 1991), which computes the values by direct summation over the rotational energy levels (Kisiel 2015; Endres et al. 2016). The maximum $J$ and $K$ values considered for the summation calculation for each conformer are detailed in the captions of the relevant tables of rotational partition functions. Further, the contributions of vibrationally excited states to the total partition function have been calculated for all vibronic groundstate conformers, and the values are tabulated alongside the rotational partition functions. The vibrational partition function values were determined by considering the anharmonic energies from our calculations, up to the vibrational states with an energy of $500 \mathrm{~cm}^{-1}$ with respect to the ground-state energy. Combining intensity information, which is provided by these rotational partition function values, with line frequencies is paramount for confirming interstellar detections and calculating column densities in the ISM.

A study such as ours, which covers a large frequency range and includes high-frequency data sets, is essential for facilitating comprehensive interstellar searches. The reason for this is twofold. First, the large frequency range allows experimental transition frequencies to be directly compared to observational spectra across a number of facilities. For example, data in the $18-26 \mathrm{GHz}$ range could be used for future searches using data from the Next Generation Very Large Array (ngVLA), where nuclear quadrupole coupling could be relevant. Similarly, the line frequencies in the $W$-band $(75-110 \mathrm{GHz})$ can be directly compared to those from ALMA Band 3 (84-116 GHz), where the hyperfine splitting has little to no effect for molecules similar in size to the amino alcohols presented here. This is particularly applicable to the vibrationally excited states we assigned in the room-temperature, highfrequency experiments. Second, our high-frequency data sets allow us to accurately define centrifugal distortion constants for
Table 5

The Rotational Partition Functions $\left(Q_{\mathrm{rot}}\right)$ for Alaninol $1, g^{\prime} G^{\prime}$ across Standard Temperatures, Implemented in the JPL Database (Pickett et al. 1998)

\begin{tabular}{lccc}
\hline \hline$T / K$ & $Q_{\text {rot }}[2 I+1=1]^{\mathrm{a}}$ & $Q_{\text {rot }}[2 I+1=3]^{\mathrm{b}}$ & $Q_{\text {vib }}$ \\
\hline 300 & 94350.6 & 283051.8 & 3.899 \\
225 & 62723.5 & 188170.5 & 2.787 \\
150 & 34638.3 & 103914.8 & 1.743 \\
75 & 12292.0 & 36876.0 & 1.090 \\
37.5 & 4347.6 & 13042.8 & 1.003 \\
18.75 & 1538.7 & 4616.2 & 1.000 \\
9.375 & 545.3 & 1635.8 & 1.000 \\
1.5 & 35.8 & 107.3 & 1.000 \\
\hline
\end{tabular}

Notes. The rotational partition functions were calculated by SPCAT (Pickett 1991) by considering $J_{\max }=220$ and $K_{\max }=40$, and they are tabulated to one decimal place. The vibrational partition function contributions $\left(Q_{\text {vib }}\right)$ were calculated by considering the theoretical anharmonic energies up to $500 \mathrm{~cm}^{-1}$. a The $2 I+1=1$ case does not consider hyperfine splitting.

b The $2 I+1=3$ case considers hyperfine splitting.

the molecules we have studied. This can, in the absence of laboratory data higher than $110 \mathrm{GHz}$, provide more accurate predictions of transition frequencies into the $\mathrm{THz}$ regime than if predictions from rotational constants from centimeter-regime data, and thus typically involving low- $J$ transitions, were simply extrapolated. The pitfalls of this were recently highlighted by Martin-Drumel et al. (2019) in their laboratory investigation and interstellar search for vinyl mercaptan. The potential issues presented there are reduced with this present study by providing high-level centrifugal distortion constants for alaninol, valinol, and leucinol.

Appendix F contains information on the machine-readable tables that are available in the electronic supplementary material. The tables contain information about the experimentally assigned transitions for each of the species assigned, as well as predictions for the rotational transitions generated from the experimentally determined rotational, centrifugal distortion, and nuclear quadrupole coupling constants for each assigned species. These predictions are consistent with the format of the - cat files produced by the SPCAT program, allowing for ease of application to astrophysical programs, such as CASSIS. ${ }^{7}$ This format also matches the format of the $\mathrm{CDMS}^{8}$ (Müller et al. 2005; Endres et al. 2016) and JPL ${ }^{9}$ (Pickett et al. 1998) databases, allowing for the archiving of this information in those databases for both the astrochemistry and molecular spectroscopy communities. It is worthy to note that the tables, in their second columns, also contain predicted line uncertainties calculated by SPCAT. These are predominantly of the order of less than $1 \mathrm{MHz}$ and are therefore useful predictions for both observational searches and further spectroscopic studies. The SPCAT program predicts all lines up to given $J$ and $K$ values, and a handful of lines in the table have larger uncertainties. These transitions are predicted within the range of the provided $J$ and $K$ values, but result from transitions with high lower state energies (generally above $120 \mathrm{~cm}^{-1}$ ) and are generally low-intensity transitions.

\footnotetext{
7 CASSIS is a software package for the analysis of astronomical spectra developed by IRAP-UPS/CNRS-http://cassis.irap.omp.eu/.

8 https://cdms.astro.uni-koeln.de/cdms/portal

9 http://spec.jpl.nasa.gov/
} 
The amino alcohols to which all of the presented predictions pertain are also attractive targets for interstellar searches due to their relatively high dipole moment components, which are essential for recording laboratory and observational rotational spectra. The components, experimentally determined for alaninol (Ellingsen et al. 1978) and theoretically calculated for valinol and leucinol, are listed in Tables 1-3. The maximum dipole moment components of the molecules are in the region of $3.0 \mathrm{D}$, which is comparable, for example, to the interstellar molecule iso-propyl cyanide (IPC), which has a maximum dipole moment component of $\mu_{a}$ around 4.0 D (Durig \& Li 1974). Other examples of similarly complex molecules have dipole moment components smaller than those of the amino alcohols. For example, aminoacetonitrile has $\mu_{a}=2.577(7) \mathrm{D}$ (Bogey et al. 1990), and hydroxyacetone has $\mu_{a}=2.22(4) \mathrm{D}$ and $\mu_{b}=2.17(2)$ D (Kattija-Ari \& Harmony 1980). This indicates that the effect due to a larger number of heavy atoms in the amino alcohols studied here can be partly compensated by the larger dipole moments. This is highlighted in a comparison of the distribution of rotational transitions for IPC and the lowest energy conformers of alaninol, valinol, and leucinol (Appendix G). The plots show a similar distribution in number and intensity of rotational transitions at $300 \mathrm{~K}$ that can be used to confirm the presence of the amino alcohols in the ISM.

\section{Conclusions}

This extensive study of the rotational spectra of alaninol, valinol, and leucinol resulted in the observation and assignment of a total of 49 species, including ground-state conformers, isotopologues in natural abundance, and low-lying vibrationally excited states. The assignments, comprising of exact transition frequencies between $2-26$ and $75-110 \mathrm{GHz}$ and rotational constants up to the quartic centrifugal distortion constants, including the nitrogen nuclear quadrupole coupling constants, are accurately reported here for the first time for the majority of the species observed. The structures of the observed amino alcohols are confirmed with a combination of isotopologue data available in natural abundance and quantum chemical calculations. We see the dominance of the intramolecular hydrogen bond in stabilizing the lowest energy conformers of each molecule, with each exhibiting similar dihedral angles between $\mathrm{N}(\mathrm{lp})-\mathrm{N}-\mathrm{C} 2-\mathrm{C} 1, \mathrm{~N}-\mathrm{C} 2-\mathrm{C} 1-\mathrm{O}$, and $\mathrm{C} 2-\mathrm{C} 1-\mathrm{O}-\mathrm{H}$.

The present work is paired with our study of the rotational spectrum of isoleucinol, which also details our $18-26 \mathrm{GHz}$ spectrometer for the first time. Together, they provide the astronomical community with the extensive data necessary to search for these astrochemically important molecules. Their detection would establish a new class of compounds observed in the ISM and could point toward areas where their amino acid analogs could be present in the gas phase.

This work has been supported by the ERC Starting Grant ASTROROT (Grant Agreement No. 638027). B.E.A. and M.F. acknowledge the International Max Planck Research School for Ultrafast Imaging and Structural Dynamics (IMPRS-UFAST). S.F. acknowledges the DESY Summer Student Program. A.L.S. acknowledges the Louise Johnson Fellowship of the
Centre for Ultrafast Imaging (CUI) at the Universität Hamburg. The authors further thank Anna Krin and Sebastién Gruet for scientific discussions.

\section{Appendix A \\ The Number of Coadded FIDs per Frequency Range per Molecule}

Table A1 is a summary of the number of coadded FIDs collected per molecule for each frequency range investigated. The experiments were typically performed until there were sufficient signal-to-noise ratios to observe and assign the ${ }^{13} \mathrm{C}$ isotopologues of the lowest energy conformer of each species.

Table A1

A Summary of the Number of Coadded FIDs, in Millions, Collected Per Molecule for Each Frequency Range Investigated

\begin{tabular}{lcccccc}
\hline \hline $\begin{array}{c}\text { Frequency } \\
\text { Region/GHz }\end{array}$ & $2-8$ & $8-13$ & $13-15.5$ & $15.5-18$ & $18-26$ & $75-110$ \\
\hline Alaninol & 1.9 & 1.1 & 1.0 & 1.4 & 2.6 & 0.5 \\
Valinol & 5.6 & 1.4 & 1.0 & 2.3 & 2.0 & 0.5 \\
Leucinol & 1.0 & 1.2 & 1.0 & 3.0 & 2.1 & 0.5 \\
\hline
\end{tabular}

\section{Appendix B \\ Nomenclature}

We introduce here the naming system used to refer to the various conformers of alaninol, valinol, and leucinol. Figure 1(a) in Section 1 contains the Lewis structures with carbon atom labels. Each structure is named with a string of letters, for example, alaninol 1 is named $g^{\prime} G g^{\prime}$, whereby each letter denotes the magnitude of a defined dihedral angle. In general, the names take the form aBcDe, where the letters refer to the following combinations: $a$ is the dihedral angle $\mathrm{N}(\mathrm{lp})-\mathrm{N}-$ $\mathrm{C} 2-\mathrm{C} 1$ (where $\mathrm{N}(\mathrm{lp})$ is the lone pair of electrons on the nitrogen atom), $\mathrm{B}$ is the $\mathrm{N}-\mathrm{C} 2-\mathrm{C} 1-\mathrm{O}$ dihedral angle, and $\mathrm{c}$ refers to the C2-C1-O-H dihedral angle (Ellingsen et al. 1978; Fausto et al. 2000). The symbols D and e take different meanings depending on the molecule being considered. $\mathrm{D}$ is the $\mathrm{C} 1-\mathrm{C} 2-\mathrm{C} 3-\mathrm{H}$ dihedral angle in valinol. In leucinol, D is the $\mathrm{C} 1-\mathrm{C} 2-\mathrm{C} 3-\mathrm{C} 4$ dihedral angle, and e is the $\mathrm{C} 2-\mathrm{C} 3-\mathrm{C} 4-\mathrm{H}$ dihedral angle. The letter used and the dihedral angle sizes they denote are $g / G$ for a dihedral angle close to $60^{\circ}$ (a gauche configuration), $g^{\prime} / G^{\prime}$ for a dihedral angle close to $-60^{\circ}$ (a gauche configuration), $t / T$ for a dihedral angle close to $180^{\circ}$ (noted as a trans configuration in Ellingsen et al. 1978 and Fausto et al. (2000), though commonly also referred to as an anti configuration).

\section{Appendix C \\ Analysis of Observed Isotopologues of Alaninol, Valinol, and Leucinol}

The experimental rotational constants for the observed isotopologues of alaninol, valinol, and leucinol are tabulated in Tables C1-C5. Values contained within square brackets were kept fixed to the values of the parent species. 
Table C1

The Experimental Rotational Constants for the Observed Isotopologues of Alaninol 1, $g^{\prime} G^{\prime}$

\begin{tabular}{|c|c|c|c|c|c|}
\hline & ${ }^{13} \mathrm{C}(1)$ & ${ }^{13} \mathrm{C}(2)$ & ${ }^{13} \mathrm{C}(3)$ & ${ }^{15} \mathrm{~N}$ & ${ }^{18} \mathrm{O}$ \\
\hline$A / \mathrm{MHz}$ & $7888.420(50)$ & $7935.239(52)$ & $7876.899(49)$ & $7715.121(56)$ & 7944.579(73) \\
\hline$B / \mathrm{MHz}$ & $3557.6341(15)$ & $3631.3380(15)$ & $3626.2698(14)$ & $3635.4848(19)$ & $3461.2597(19)$ \\
\hline$C / \mathrm{MHz}$ & 2693.2494(13) & $2744.2287(13)$ & $2732.7080(13)$ & 2716.7871(16) & $2644.4232(20)$ \\
\hline$\Delta_{J} / \mathrm{kHz}$ & [0.72345] & [0.72345] & [0.72345] & [0.72345] & [0.72345] \\
\hline$\Delta_{J K} / \mathrm{kHz}$ & [3.4438] & [3.4438] & [3.4438] & [3.4438] & [3.4438] \\
\hline$\Delta_{K} / \mathrm{kHz}$ & [2.024] & [2.024] & {$[2.024]$} & [2.024] & [2.024] \\
\hline$\delta_{J} / \mathrm{kHz}$ & [0.17391] & [0.17391] & [0.17391] & [0.17391] & {$[0.17391]$} \\
\hline$\delta_{K} / \mathrm{kHz}$ & [2.321] & [2.321] & [2.321] & {$[2.321]$} & [2.321] \\
\hline$\chi_{a a} / \mathrm{MHz}$ & $-3.723(9)$ & $-3.671(9)$ & $-3.646(8)$ & $\ldots$ & $-3.677(15)$ \\
\hline$\chi_{b b}-\chi_{c c} / \mathrm{MHz}$ & $-0.0560(224)$ & $-0.1080(284)$ & $-0.1600(236)$ & $\cdots$ & $-0.0720(376)$ \\
\hline No. of lines & 44 & 43 & 44 & 14 & 38 \\
\hline$\sigma / \mathrm{kHz}$ & 13.8 & 14.1 & 13.8 & 9.4 & 18.5 \\
\hline$J_{\max }, K_{\max }$ & 4,4 & 4,4 & 4,4 & 4,4 & 4,4 \\
\hline
\end{tabular}

Table C2

The Experimental Rotational Constants for the Observed Isotopologues of Alaninol 2, $g G^{\prime} g$

\begin{tabular}{|c|c|c|c|c|}
\hline & ${ }^{13} \mathrm{C}(1)$ & 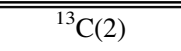 & ${ }^{{ }^{13} \mathrm{C}(3)}$ & ${ }^{15} \mathrm{~N}$ \\
\hline$A / \mathrm{MHz}$ & $6348.5973(39)$ & $6442.0197(19)$ & $6421.5158(19)$ & $6309.604(66)$ \\
\hline$B / \mathrm{MHz}$ & $4095.5493(32)$ & $4124.3613(17)$ & $4100.6636(16)$ & $4116.8509(40)$ \\
\hline$C / \mathrm{MHz}$ & $3277.8513(27)$ & $3328.5900(14)$ & $3317.8295(14)$ & $3289.5738(46)$ \\
\hline$\Delta_{J} / \mathrm{kHz}$ & [1.6343] & [1.6343] & [1.6343] & [1.6343] \\
\hline$\Delta_{J K} / \mathrm{kHz}$ & [4.940] & [4.940] & [4.940] & [4.940] \\
\hline$\Delta_{K} / \mathrm{kHz}$ & {$[-3.701]$} & {$[-3.701]$} & {$[-3.701]$} & {$[-3.701]$} \\
\hline$\delta_{J} / \mathrm{kHz}$ & [0.24994] & [0.24994] & [0.24994] & [0.24994] \\
\hline$\delta_{K} / \mathrm{kHz}$ & [1.115] & [1.115] & [1.115] & {$[1.115]$} \\
\hline$\chi_{a a} / \mathrm{MHz}$ & $-0.685(20)$ & $-0.705(14)$ & $-0.649(13)$ & $\ldots$ \\
\hline$\chi_{b b}-\chi_{c c} / \mathrm{MHz}$ & $4.068(72)$ & $4.0384(268)$ & $4.0880(260)$ & $\cdots$ \\
\hline No. of lines & 21 & 37 & 32 & 5 \\
\hline$\sigma / \mathrm{kHz}$ & 20.3 & 17.4 & 15.3 & 3.6 \\
\hline$J_{\max }, K_{\max }$ & 4,4 & 3,3 & 3,3 & 3,3 \\
\hline
\end{tabular}

Table C3

The Experimental Rotational Constants for the Observed Isotopologues of Valinol $1, g^{\prime} G^{\prime} G^{\prime}$

\begin{tabular}{lccccccc}
\hline \hline & ${ }^{13} \mathrm{C}(1)$ & ${ }^{13} \mathrm{C}(2)$ & ${ }^{13} \mathrm{C}(3)$ & ${ }^{13} \mathrm{C}(4)$ & ${ }^{13} \mathrm{C}(5)$ & ${ }^{15} \mathrm{~N}$ \\
\hline$A / \mathrm{MHz}$ & $4020.1232(14)$ & $4020.4398(18)$ & $4004.2223(11)$ & $3948.5415(15)$ & $3999.9141(14)$ & $3946.0314(33)$ & $4013.470(74)$ \\
$B / \mathrm{MHz}$ & $1692.8142(46)$ & $1700.92434(56)$ & $1691.58057(49)$ & $1686.74869(48)$ & $1672.71042(54)$ & $1698.5943(10)$ & $1629.6483(14)$ \\
$C / \mathrm{MHz}$ & $1279.64920(35)$ & $1283.99785(42)$ & $1277.09091(38)$ & $1268.26992(38)$ & $1265.72637(36)$ & $1274.83000(51)$ & $1242.5404(12)$ \\
$\Delta_{J} / \mathrm{kHz}$ & {$[0.108023]$} & {$[0.108023]$} & {$[0.108023]$} & {$[0.108023]$} & {$[0.108023]$} & {$[0.108023]$} & {$[0.108023]$} \\
$\Delta_{J K} / \mathrm{kHz}$ & {$[0.18277]$} & {$[0.18277]$} & {$[0.18277]$} & {$[0.18277]$} & {$[0.18277]$} & {$[0.18277]$} & {$[0.18277]$} \\
$\delta_{J} / \mathrm{kHz}$ & {$[0.026377]$} & {$[0.026377]$} & {$[0.026377]$} & {$[0.026377]$} & {$[0.026377]$} & {$[0.026377]$} & {$[0.026377]$} \\
$\chi_{a a} / \mathrm{MHz}$ & $-4.157(10)$ & $-4.200(17)$ & $-4.178(10)$ & $-4.189(15)$ & $-1.391(9)$ & $\ldots$ & $-4.213(31)$ \\
$\chi_{b b}-\chi_{c c} / \mathrm{MHz}$ & $0.7020(276)$ & $0.668(60)$ & $0.6668(352)$ & $0.696(44)$ & $0.7236(304)$ & $\ldots$ & $0.092(392)$ \\
No. of lines & 69 & 55 & 56 & 71 & 15.6 & 13.1 & 19 \\
$\sigma / \mathrm{kHz}$ & 13.7 & 16.8 & 12.6 & 9,9 & 9,9 & 14.6 & 9,9 \\
$J_{\max }, K_{\max }$ & 9,9 & 9,9 & 9,9 & & 9,9 \\
\hline
\end{tabular}


Table C4

The Experimental Rotational Constants for the Observed Isotopologues of Valinol 2, $g^{\prime} G g^{\prime} G$

\begin{tabular}{|c|c|c|c|c|c|c|}
\hline & ${ }^{13} \mathrm{C}(1)$ & ${ }^{13} \mathrm{C}(2)$ & ${ }^{13} \mathrm{C}(3)$ & ${ }^{13} \mathrm{C}(4)$ & ${ }^{13} \mathrm{C}(5)$ & $\overline{{ }^{15} \mathrm{~N}}$ \\
\hline$A / \mathrm{MHz}$ & $4217.25(26)$ & $4230.96(16)$ & $4227.82(18)$ & $4203.53(14)$ & $4165.46(23)$ & 4161.13(46) \\
\hline$B / \mathrm{MHz}$ & $1537.6160(10)$ & $1546.15373(94)$ & $1539.5664(10)$ & $1521.8131(10)$ & $1532.9518(10)$ & $1541.7806(52)$ \\
\hline $\mathrm{C} / \mathrm{MHz}$ & $1404.8932(10)$ & $1413.57389(91)$ & $1408.5188(10)$ & $1392.5804(10)$ & $1398.6118(10)$ & $1408.0969(39)$ \\
\hline$\Delta_{J K} / \mathrm{kHz}$ & [0.12889] & [0.12889] & [0.12889] & [0.12889] & [0.12889] & [0.12889] \\
\hline$\Delta_{K} / \mathrm{kHz}$ & [1.039662972] & [1.039662972] & [1.039662972] & [1.039662972] & [1.039662972] & [1.039662972] \\
\hline$\delta_{J} / \mathrm{kHz}$ & [0.043245] & [0.043245] & [0.043245] & [0.043245] & [0.043245] & [0.043245] \\
\hline$\chi_{b b}-\chi_{c c} / \mathrm{MHz}$ & {$[-0.6612]$} & {$[-0.6612]$} & {$[-0.6612]$} & {$[-0.6612]$} & {$[-0.6612]$} & $\cdots$ \\
\hline No. of lines & 17 & 20 & 13 & 24 & 14 & 7 \\
\hline$\sigma / \mathrm{kHz}$ & 13.1 & 11.4 & 12.0 & 12.4 & 12.0 & 17.2 \\
\hline$J_{\max }, K_{\max }$ & 8,8 & 8,8 & 8,8 & 9,9 & 8,8 & 8,8 \\
\hline
\end{tabular}

Table C5

The Experimental Rotational Constants for the Observed Isotopologues of Leucinol $1, g^{\prime} G g^{\prime} T g$

\begin{tabular}{|c|c|c|c|c|c|c|c|}
\hline & ${ }^{13} \mathrm{C}(1)$ & ${ }^{13} \mathrm{C}(2)$ & ${ }^{13} \mathrm{C}(3)$ & ${ }^{13} \mathrm{C}(4)$ & ${ }^{13} \mathrm{C}(5)$ & ${ }^{13} \mathrm{C}(6)$ & ${ }^{15} \mathrm{~N}$ \\
\hline$A / \mathrm{MHz}$ & $4025.64(40)$ & 4046.97(36) & $4032.59(38)$ & $4046.55(44)$ & $4022.16(48)$ & 3984.01(43) & $3980.24(49)$ \\
\hline$B / \mathrm{MHz}$ & $898.26547(49)$ & $903.73248(43)$ & $904.24474(46)$ & $899.37101(47)$ & $890.18423(44)$ & $896.39878(41)$ & $900.95139(47)$ \\
\hline$C / \mathrm{MHz}$ & $870.06898(50)$ & $875.96767(42)$ & $875.77149(45)$ & $871.91906(43)$ & $862.41534(44)$ & $868.61992(40)$ & $873.73532(49)$ \\
\hline$\Delta_{J} / \mathrm{kHz}$ & $0.07430(98)$ & $0.06707(84)$ & $0.07240(90)$ & $0.07210(88)$ & $0.06907(87)$ & $0.07393(78)$ & $0.0717(10)$ \\
\hline$\Delta_{J K} / \mathrm{kHz}$ & $0.418(14)$ & [0.4674] & $0.421(21)$ & $0.452(17)$ & $0.524(26)$ & $0.408(11)$ & $0.390(19)$ \\
\hline$\Delta_{K} / \mathrm{kHz}$ & [1.198] & [1.198] & [1.198] & [1.198] & [1.198] & [1.198] & [1.198] \\
\hline$\delta_{J} / \mathrm{kHz}$ & {$[0.01315]$} & {$[0.01315]$} & {$[0.01315]$} & {$[0.01315]$} & {$[0.01315]$} & {$[0.01315]$} & {$[0.01315]$} \\
\hline$\delta_{K} / \mathrm{kHz}$ & [7.583] & [7.583] & [7.583] & [7.583] & [7.583] & [7.583] & [7.583] \\
\hline$\chi_{a a} / \mathrm{MHz}$ & {$[-3.8315]$} & {$[-3.8315]$} & {$[-3.8315]$} & {$[-3.8315]$} & {$[-3.8315]$} & {$[-3.8315]$} & $\ldots$ \\
\hline$\chi_{b b}-\chi_{c c} / \mathrm{MHz}$ & {$[-1.0472]$} & {$[-1.0472]$} & {$[-1.0472]$} & {$[-1.0472]$} & {$[-1.0472]$} & {$[-1.0472]$} & cdots \\
\hline No. of lines & 64 & 48 & 60 & 57 & 54 & 57 & 35 \\
\hline$\sigma / \mathrm{kHz}$ & 15.7 & 10.7 & 13.5 & 13.3 & 13.4 & 11.3 & 11.0 \\
\hline$J_{\max }, K_{\max }$ & 14,14 & 14,14 & 14,14 & 14,13 & 14,14 & 14,13 & 14,13 \\
\hline
\end{tabular}

\section{Appendix D \\ Vibrationally Excited States of Alaninol, Valinol, and Leucinol}

The motions of the fundamental vibrational modes of alaninol 1, $g^{\prime} G g^{\prime}$, are described in Section 5. The relative energies of the fundamental modes, as well as a depiction of the main motions, are shown in Figure D1. The rotational constants of the assigned vibrationally excited state of alaninol $2, g G^{\prime} g$, are provided in Table D1.

The rotational spectra of three fundamental vibrational modes of valinol $1, g^{\prime} G g^{\prime} G^{\prime}$, were assigned in this work. The rotational constants for these states are shown in Table D2. The $\nu_{54}$ fundamental mode of valinol $1, g^{\prime} G g^{\prime} G^{\prime}$, is a carbon backbone twist. The $\nu_{53}$ mode is an asymmetric rocking motion centered on the $\mathrm{C} 1$ nucleus. The third state is the overtone of $\nu_{54}$.

The vibrational modes and their associated energies, as obtained from the anharmonic frequency calculations, are presented in Tables D3-D11. The calculations were all performed at the B3LYP-D3(BJ)/aug-cc-pVTZ level of theory; see main text (Section 3) for details. The modes up to $500 \mathrm{~cm}^{-1}$ (based on anharmonic energies) are included, and their harmonic $\left(E^{h}\right)$ and anharmonic $\left(E^{a}\right)$ energies are listed to one decimal place.
Table D1

The Experimental and Theoretical Rotational Constants for the Observed Vibrational State of Alaninol 2, $g G^{\prime} g$

\begin{tabular}{|c|c|c|}
\hline \multirow{4}{*}{$\begin{array}{l}E^{h} / \mathrm{cm}^{-1}\left[\mathrm{~kJ} \mathrm{~mol}^{-1}\right] \\
E^{a} / \mathrm{cm}^{-1}\left[\mathrm{~kJ} \mathrm{~mol}^{-1}\right]\end{array}$} & \multicolumn{2}{|c|}{$\nu_{36}$} \\
\hline & \multicolumn{2}{|c|}{$141.4[1.7]$} \\
\hline & \multicolumn{2}{|c|}{$139.1[1.7]$} \\
\hline & This Work & Theory \\
\hline$A / \mathrm{MHz}$ & $6468.737(31)$ & 6469.0 \\
\hline$B / \mathrm{MHz}$ & $4138.1980(31)$ & 4136.8 \\
\hline$C / \mathrm{MHz}$ & $3323.3733(34)$ & 3322.2 \\
\hline$\Delta_{J} / \mathrm{kHz}$ & $1.6329(51)$ & \\
\hline$\Delta_{J K} / \mathrm{kHz}$ & $5.433(29)$ & \\
\hline$\Delta_{K} / \mathrm{kHz}$ & $-4.28(72)$ & \\
\hline$\delta_{J} / \mathrm{kHz}$ & $0.2583(34)$ & \\
\hline$\delta_{K} / \mathrm{kHz}$ & $1.333(84)$ & \\
\hline No. of lines & 46 & \\
\hline$\sigma / \mathrm{kHz}$ & 28.5 & \\
\hline
\end{tabular}

Note. The theoretical parameters were obtained at the B3LYP-D3(BJ)/aug-ccpVTZ level of theory and shifted by the following values to account for the differences in the experimental and theoretical rotational constants of the vibronic ground state: $\Delta A=35.6 \mathrm{MHz}, \Delta B=67.8 \mathrm{MHz}, \Delta C=57.9 \mathrm{MHz}$. The harmonic and anharmonic energies $\left(E^{h}\right.$ and $E^{a}$, respectively) of the vibrational states are relative to the energy of the alaninol $2, g G^{\prime} g$, conformer. 


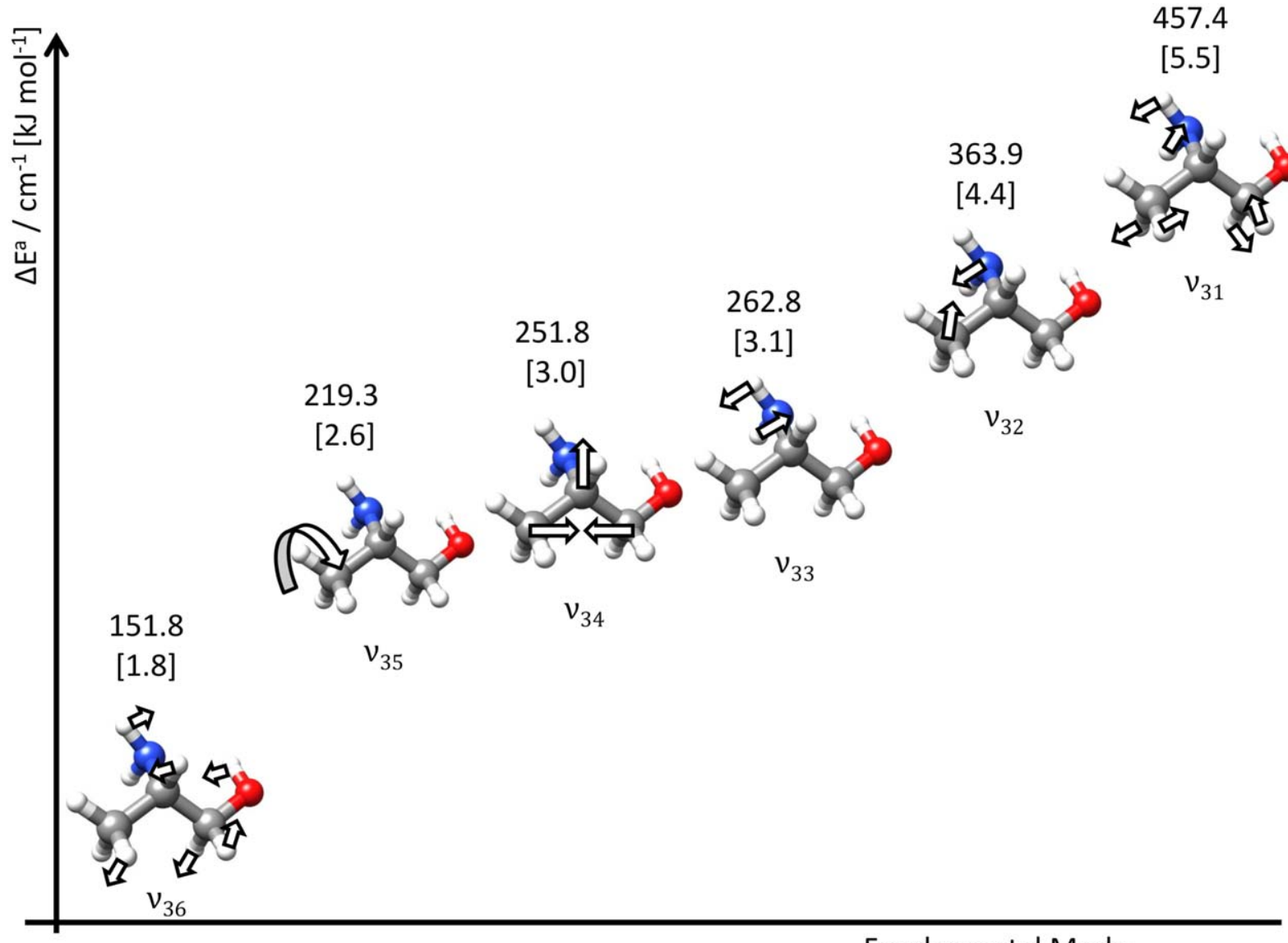

Fundamental Mode

Figure D1. A summary of the assigned fundamental vibrations of alaninol $1, g^{\prime} G g^{\prime}$. The predominant motion for each mode is indicated by the arrows; a description can be found in the main text. The anharmonic energies presented are those calculated at the B3LYP-D3(BJ)/aug-cc-pVTZ level of theory.

Table D2

The Experimental and Theoretical Rotational Constants for the Observed Vibrational States of Valinol $1, g^{\prime} G g^{\prime} G^{\prime}$

\begin{tabular}{|c|c|c|c|c|c|c|}
\hline \multirow{4}{*}{$\begin{array}{l}E^{h} / \mathrm{cm}^{-1}\left[\mathrm{~kJ} \mathrm{~mol}^{-1}\right] \\
E^{a} / \mathrm{cm}^{-1}\left[\mathrm{~kJ} \mathrm{~mol}^{-1}\right]\end{array}$} & \multicolumn{2}{|c|}{$\nu_{54}$} & \multicolumn{2}{|c|}{$\nu_{53}$} & \multicolumn{2}{|c|}{$2 \nu_{54}$} \\
\hline & \multicolumn{2}{|c|}{$74.1[0.9]$} & \multicolumn{2}{|c|}{$124.3[1.5]$} & \multicolumn{2}{|c|}{$148.2[1.8]$} \\
\hline & \multicolumn{2}{|c|}{$69.7[0.8]$} & \multicolumn{2}{|c|}{$117.7[1.4]$} & \multicolumn{2}{|c|}{$139.3[1.7]$} \\
\hline & This Work & Theory & This Work & Theory & This Work & Theory \\
\hline$A / \mathrm{MHz}$ & $4020.001(11)$ & 4019.3 & 4017.841(12) & 4008.5 & 4009.515(11) & 4015.1 \\
\hline$B / \mathrm{MHz}$ & $1697.89013(67)$ & 1698.1 & $1694.6255(21)$ & 1702.2 & 1694.6859(12) & 1694.9 \\
\hline$C / \mathrm{MHz}$ & $1284.60788(48)$ & 1284.8 & $1285.09357(59)$ & 1284.8 & $1285.22224(98)$ & 1285.3 \\
\hline$\Delta_{J} / \mathrm{kHz}$ & $0.10835(25)$ & $\cdots$ & $0.10933(61)$ & $\cdots$ & $0.11503(28)$ & $\ldots$ \\
\hline$\Delta_{J K} / \mathrm{kHz}$ & $0.1909(11)$ & $\ldots$ & $0.2163(16)$ & $\ldots$ & $0.15858(80)$ & $\cdots$ \\
\hline$\Delta_{K} / \mathrm{kHz}$ & $0.738(78)$ & $\ldots$ & [0.724] & $\ldots$ & [0.724] & $\cdots$ \\
\hline$\delta_{J} / \mathrm{kHz}$ & $0.02641(13)$ & $\cdots$ & $0.02709(36)$ & $\ldots$ & $0.02221(23)$ & $\ldots$ \\
\hline$\delta_{K} / \mathrm{kHz}$ & $0.2643(26)$ & $\cdots$ & {$[0.2496]$} & $\cdots$ & {$[0.2496]$} & $\cdots$ \\
\hline No. of lines & 100 & $\cdots$ & 31 & $\cdots$ & 34 & $\cdots$ \\
\hline$\sigma / \mathrm{kHz}$ & 25.9 & $\ldots$ & 25.0 & $\ldots$ & 27.6 & $\ldots$ \\
\hline
\end{tabular}

Note. The theoretical parameters were obtained at the B3LYP-D3(BJ)/aug-cc-pVTZ level of theory and shifted by the following values to account for the differences in the experimental and theoretical rotational constants of the vibronic ground state: $\Delta A=31.0 \mathrm{MHz}, \Delta B=18.0 \mathrm{MHz}, \Delta C=14.5 \mathrm{MHz}$. The harmonic and anharmonic energies ( $E^{h}$ and $E^{a}$ respectively) of the vibrational states are relative to the energy of the valinol $1, g^{\prime} G g^{\prime} G^{\prime}$, conformer. Distortion constants contained within square brackets were kept fixed to the values of the parent species. 
Table D3

Vibrationally Excited States of Alaninol $1, g^{\prime} G g^{\prime}$, that Reside under $500 \mathrm{~cm}^{-1}$ in Energy Relative to the Ground State

\begin{tabular}{lrr}
\hline \hline Vibrational Mode & $E^{h} / \mathrm{cm}^{-1}$ & $E^{a} / \mathrm{cm}^{-1}$ \\
\hline$\nu_{36}$ & 146.3 & 151.8 \\
$\nu_{35}$ & 228.7 & 219.3 \\
$\nu_{34}$ & 257.4 & 251.8 \\
$\nu_{33}$ & 266.5 & 262.8 \\
$2 \nu_{36}$ & 292.7 & 306.1 \\
$\nu_{32}$ & 365.2 & 363.9 \\
$\nu_{36}+\nu_{35}$ & 375.0 & 375.0 \\
$\nu_{36}+\nu_{34}$ & 403.7 & 400.9 \\
$\nu_{36}+\nu_{33}$ & 412.9 & 413.2 \\
$2 \nu_{35}$ & 457.4 & 425.9 \\
$\nu_{31}$ & 451.4 & 457.4 \\
$\nu_{35}+\nu_{34}$ & 486.1 & 470.2 \\
$\nu_{35}+\nu_{33}$ & 495.2 & 479.6 \\
$\nu_{29}$ & 550.0 & 493.8 \\
$2 \nu_{34}$ & 514.7 & 496.6 \\
\hline
\end{tabular}

Table D4

Vibrationally Excited States of Alaninol 2, $g G^{\prime} g$, that Reside under $500 \mathrm{~cm}^{-1}$ in Energy Relative to the Ground State

\begin{tabular}{lrr}
\hline \hline Vibrational Mode & $E^{h} / \mathrm{cm}^{-1}$ & $E^{a} / \mathrm{cm}^{-1}$ \\
\hline$\nu_{36}$ & 141.4 & 139.1 \\
$\nu_{35}$ & 214.7 & 208.2 \\
$\nu_{34}$ & 259.4 & 248.3 \\
$\nu_{33}$ & 283.2 & 265.2 \\
$2 \nu_{36}$ & 282.8 & 282.1 \\
$\nu_{36}+\nu_{35}$ & 356.0 & 346.7 \\
$\nu_{32}$ & 375.6 & 373.8 \\
$\nu_{36}+\nu_{34}$ & 400.8 & 383.7 \\
$\nu_{31}$ & 411.2 & 406.0 \\
$\nu_{36}+\nu_{33}$ & 424.5 & 406.6 \\
$2 \nu_{35}$ & 429.3 & 408.4 \\
$\nu_{35}+\nu_{34}$ & 474.1 & 458.1 \\
$\nu_{30}$ & 529.9 & 461.5 \\
$\nu_{35}+\nu_{33}$ & 497.8 & 470.5 \\
$2 \nu_{34}$ & 518.8 & 493.3 \\
\hline
\end{tabular}

Table D5

Vibrationally Excited States of Valinol $1, g^{\prime} G^{\prime} G^{\prime}$, that Reside under $500 \mathrm{~cm}^{-1}$ in Energy Relative to the Ground State

\begin{tabular}{lrr}
\hline \hline Vibrational Mode & $E^{h} / \mathrm{cm}^{-1}$ & $E^{a} / \mathrm{cm}^{-1}$ \\
\hline$\nu_{54}$ & 74.1 & 69.7 \\
$\nu_{53}$ & 124.3 & 117.7 \\
$2 \nu_{54}$ & 148.2 & 139.3 \\
$\nu_{54}+\nu_{53}$ & 198.4 & 188.5 \\
$\nu_{52}$ & 190.4 & 195.7 \\
$\nu_{49}$ & 269.2 & 198.4 \\
$\nu_{50}$ & 240.7 & 226.4 \\
$2 \nu_{53}$ & 248.6 & 236.6 \\
$\nu_{51}$ & 231.6 & 240.5 \\
$\nu_{54}+\nu_{49}$ & 343.4 & 260.9 \\
$\nu_{54}+\nu_{52}$ & 264.5 & 268.9 \\
$\nu_{54}+\nu_{50}$ & 314.8 & 294.1 \\
$\nu_{48}$ & 312.2 & 299.1 \\
$\nu_{53}+\nu_{49}$ & 393.5 & 308.8 \\
$\nu_{54}+\nu_{51}$ & 312.3 \\
$\nu_{53}+\nu_{52}$ & 305.7 & 314.5 \\
$\nu_{47}$ & 314.7 & 338.3 \\
$\nu_{53}+\nu_{50}$ & 356.0 & 339.5 \\
$\nu_{53}+\nu_{51}$ & 365.0 & 358.8
\end{tabular}

Table D5

(Continued)

\begin{tabular}{lrr}
\hline \hline Vibrational Mode & $E^{h} / \mathrm{cm}^{-1}$ & $E^{a} / \mathrm{cm}^{-1}$ \\
\hline$\nu_{54}+\nu_{48}$ & 386.4 & 368.1 \\
$2 \nu_{49}$ & 538.5 & 368.5 \\
$\nu_{46}$ & 375.9 & 374.1 \\
$\nu_{52}+\nu_{49}$ & 459.6 & 392.4 \\
$2 \nu_{52}$ & 380.7 & 393.3 \\
$\nu_{54}+\nu_{47}$ & 430.1 & 406.3 \\
$\nu_{53}+\nu_{48}$ & 436.5 & 415.5 \\
$\nu_{45}$ & 427.7 & 416.7 \\
$\nu_{52}+\nu_{50}$ & 431.0 & 420.4 \\
$\nu_{50}+\nu_{49}$ & 509.9 & 426.1 \\
$\nu_{51}+\nu_{49}$ & 500.8 & 434.1 \\
$\nu_{52}+\nu_{51}$ & 422.0 & 439.7 \\
$2 \nu_{50}$ & 481.3 & 443.9 \\
$\nu_{54}+\nu_{46}$ & 450.0 & 446.0 \\
$\nu_{53}+\nu_{47}$ & 480.3 & 454.9 \\
$\nu_{51}+\nu_{50}$ & 472.3 & 468.1 \\
$\nu_{44}$ & 483.8 & 480.0 \\
$2 \nu_{51}$ & 463.2 & 480.2 \\
$\nu_{54}+\nu_{45}$ & 501.8 & 483.9 \\
$\nu_{49}+\nu_{48}$ & 581.5 & 489.6 \\
$\nu_{52}+\nu_{48}$ & 502.6 & 493.0 \\
$\nu_{54}+\nu_{46}$ & 500.2 & 493.7 \\
\hline
\end{tabular}

Table D6

Vibrationally Excited States of Valinol 2, $g^{\prime} G g^{\prime} G$, that Reside under $500 \mathrm{~cm}^{-1}$ in Energy Relative to the Ground State

\begin{tabular}{lrr}
\hline \hline Vibrational Mode & $E^{h} / \mathrm{cm}^{-1}$ & $E^{a} / \mathrm{cm}^{-1}$ \\
\hline$\nu_{54}$ & 65.9 & 87.0 \\
$\nu_{53}$ & 124.1 & 123.7 \\
$2 \nu_{54}$ & 131.9 & 181.1 \\
$\nu_{52}$ & 201.2 & 195.2 \\
$\nu_{54}+\nu_{53}$ & 190.0 & 219.4 \\
$\nu_{51}$ & 221.7 & 224.0 \\
$\nu_{50}$ & 240.4 & 231.4 \\
$2 \nu_{53}$ & 248.2 & 246.8 \\
$\nu_{49}$ & 269.1 & 264.0 \\
$\nu_{48}$ & 290.9 & 286.7 \\
$\nu_{54}+\nu_{52}$ & 267.1 & 288.9 \\
$\nu_{54}+\nu_{51}$ & 287.6 & 317.4 \\
$\nu_{54}+\nu_{50}$ & 306.4 & 319.1 \\
$\nu_{53}+\nu_{52}$ & 325.3 & 323.2 \\
$\nu_{47}$ & 326.2 & 324.3 \\
$\nu_{46}$ & 340.6 & 339.1 \\
$\nu_{53}+\nu_{51}$ & 345.8 & 348.5 \\
$\nu_{54}+\nu_{49}$ & 335.0 & 351.9 \\
$\nu_{53}+\nu_{50}$ & 364.5 & 356.2 \\
$\nu_{54}+\nu_{48}$ & 356.8 & 376.3 \\
$\nu_{53}+\nu_{49}$ & 393.2 & 384.5 \\
$2 \nu_{52}$ & 402.4 & 400.3 \\
$\nu_{10}$ & 3024.4 & 402.5 \\
$\nu_{54}+\nu_{47}$ & 392.1 & 411.4 \\
$\nu_{53}+\nu_{48}$ & 415.0 & 412.8 \\
$\nu_{52}+\nu_{51}$ & 422.9 & 423.2 \\
$\nu_{54}+\nu_{46}$ & 406.6 & 424.6 \\
$\nu_{52}+\nu_{50}$ & 441.7 & 439.2 \\
$\nu_{45}$ & 447.1 & 447.9 \\
$\nu_{51}+\nu_{50}$ & 462.1 & 458.0 \\
$2 \nu_{51}$ & 443.3 & \\
$\nu_{53}+\nu_{47}$ & 450.3 & \\
$2 \nu_{50}$ & 480.9 & \\
$\nu_{53}+\nu_{46}$ & 464.8 & \\
& & \\
& & \\
& &
\end{tabular}


Table D6

(Continued)

\begin{tabular}{lcc}
\hline \hline Vibrational Mode & $E^{h} / \mathrm{cm}^{-1}$ & $E^{a} / \mathrm{cm}^{-1}$ \\
\hline$\nu_{52}+\nu_{49}$ & 470.3 & 464.5 \\
$\nu_{43}$ & 544.4 & 485.1 \\
$\nu_{44}$ & 496.7 & 486.3 \\
$\nu_{52}+\nu_{48}$ & 492.1 & 488.7 \\
$\nu_{51}+\nu_{49}$ & 490.8 & 489.7 \\
$\nu_{50}+\nu_{49}$ & 509.5 & 495.1 \\
\hline
\end{tabular}

Table D7

Vibrationally Excited States of Valinol 3, $g^{\prime} G g^{\prime} T$, that Reside under $500 \mathrm{~cm}^{-1}$ in Energy Relative to the Ground State

\begin{tabular}{|c|c|c|}
\hline Vibrational Mode & $E^{h} / \mathrm{cm}^{-1}$ & $E^{a} / \mathrm{cm}^{-1}$ \\
\hline$\nu_{54}$ & 74.7 & 43.0 \\
\hline $2 \nu_{54}$ & 149.4 & 81.1 \\
\hline$\nu_{53}$ & 122.3 & 101.8 \\
\hline$\nu_{54}+\nu_{53}$ & 197.0 & 139.4 \\
\hline $2 \nu_{53}$ & 244.6 & 202.5 \\
\hline$\nu_{52}$ & 215.5 & 207.8 \\
\hline$\nu_{51}$ & 231.5 & 213.5 \\
\hline$\nu_{50}$ & 245.5 & 230.7 \\
\hline$\nu_{54}+\nu_{52}$ & 290.2 & 252.5 \\
\hline$\nu_{49}$ & 263.1 & 255.3 \\
\hline$\nu_{54}+\nu_{51}$ & 306.2 & 255.5 \\
\hline$\nu_{48}$ & 277.7 & 256.4 \\
\hline$\nu_{54}+\nu_{50}$ & 320.2 & 276.1 \\
\hline$\nu_{54}+\nu_{49}$ & 337.8 & 293.5 \\
\hline$\nu_{54}+\nu_{48}$ & 352.4 & 295.7 \\
\hline$\nu_{53}+\nu_{52}$ & 337.8 & 309.0 \\
\hline$\nu_{53}+\nu_{51}$ & 353.8 & 312.2 \\
\hline$\nu_{47}$ & 321.5 & 316.7 \\
\hline$\nu_{53}+\nu_{50}$ & 367.8 & 331.2 \\
\hline$\nu_{46}$ & 351.4 & 348.6 \\
\hline$\nu_{53}+\nu_{49}$ & 385.4 & 353.7 \\
\hline$\nu_{53}+\nu_{48}$ & 400.0 & 355.4 \\
\hline$\nu_{54}+\nu_{47}$ & 396.2 & 360.6 \\
\hline$\nu_{54}+\nu_{46}$ & 426.2 & 390.5 \\
\hline $2 \nu_{52}$ & 431.0 & 414.3 \\
\hline$\nu_{53}+\nu_{47}$ & 443.8 & 418.5 \\
\hline$\nu_{52}+\nu_{51}$ & 447.0 & 420.2 \\
\hline $2 \nu_{51}$ & 463.0 & 422.4 \\
\hline$\nu_{51}+\nu_{50}$ & 477.0 & 429.8 \\
\hline$\nu_{52}+\nu_{50}$ & 461.0 & 435.5 \\
\hline$\nu_{53}+\nu_{46}$ & 473.7 & 449.8 \\
\hline $2 \nu_{50}$ & 491.1 & 456.0 \\
\hline$\nu_{45}$ & 463.5 & 459.1 \\
\hline$\nu_{52}+\nu_{49}$ & 478.6 & 462.4 \\
\hline$\nu_{52}+\nu_{48}$ & 493.2 & 463.3 \\
\hline$\nu_{51}+\nu_{48}$ & 509.2 & 468.5 \\
\hline$\nu_{51}+\nu_{49}$ & 494.6 & 468.6 \\
\hline$\nu_{43}$ & 550.7 & 481.4 \\
\hline$\nu_{44}$ & 500.0 & 484.2 \\
\hline$\nu_{50}+\nu_{49}$ & 508.6 & 485.4 \\
\hline$\nu_{50}+\nu_{48}$ & 523.2 & 486.0 \\
\hline
\end{tabular}

Table D8

Vibrationally Excited States of Valinol $4, g G^{\prime} g G^{\prime}$, that Reside under $500 \mathrm{~cm}^{-1}$ in Energy Relative to the Ground State

\begin{tabular}{|c|c|c|}
\hline Vibrational Mode & $\overline{E^{h} / \mathrm{cm}^{-1}}$ & $E^{a} / \mathrm{cm}^{-1}$ \\
\hline$\nu_{54}$ & 47.1 & 54.9 \\
\hline $2 \nu_{54}$ & 94.2 & 110.1 \\
\hline$\nu_{53}$ & 122.2 & 112.7 \\
\hline$\nu_{54}+\nu_{53}$ & 169.3 & 169.1 \\
\hline$\nu_{52}$ & 197.9 & 197.7 \\
\hline $2 \nu_{53}$ & 244.3 & 223.1 \\
\hline$\nu_{51}$ & 227.3 & 224.2 \\
\hline$\nu_{50}$ & 242.7 & 244.8 \\
\hline$\nu_{54}+\nu_{52}$ & 245.0 & 258.8 \\
\hline$\nu_{49}$ & 274.9 & 259.4 \\
\hline$\nu_{54}+\nu_{51}$ & 274.4 & 281.2 \\
\hline$\nu_{48}$ & 305.8 & 297.3 \\
\hline$\nu_{54}+\nu_{50}$ & 289.8 & 299.2 \\
\hline$\nu_{53}+\nu_{52}$ & 320.0 & 311.4 \\
\hline$\nu_{54}+\nu_{49}$ & 322.0 & 323.9 \\
\hline$\nu_{53}+\nu_{51}$ & 349.4 & 336.9 \\
\hline$\nu_{53}+\nu_{50}$ & 364.9 & 356.8 \\
\hline$\nu_{54}+\nu_{48}$ & 352.9 & 358.4 \\
\hline$\nu_{53}+\nu_{49}$ & 397.1 & 371.6 \\
\hline$\nu_{47}$ & 383.4 & 377.2 \\
\hline$\nu_{46}$ & 394.4 & 392.5 \\
\hline $2 \nu_{52}$ & 395.7 & 398.5 \\
\hline$\nu_{53}+\nu_{48}$ & 427.9 & 412.5 \\
\hline$\nu_{45}$ & 416.9 & 413.4 \\
\hline$\nu_{52}+\nu_{51}$ & 425.1 & 417.7 \\
\hline$\nu_{54}+\nu_{47}$ & 430.5 & 430.9 \\
\hline$\nu_{52}+\nu_{50}$ & 440.5 & 438.7 \\
\hline $2 \nu_{51}$ & 454.5 & 444.6 \\
\hline$\nu_{54}+\nu_{46}$ & 441.5 & 447.8 \\
\hline$\nu_{44}$ & 461.6 & 456.3 \\
\hline$\nu_{52}+\nu_{49}$ & 472.8 & 457.1 \\
\hline$\nu_{51}+\nu_{50}$ & 470.0 & 466.4 \\
\hline$\nu_{54}+\nu_{45}$ & 464.0 & 468.0 \\
\hline$\nu_{43}$ & 527.6 & 469.6 \\
\hline$\nu_{51}+\nu_{49}$ & 502.2 & 483.5 \\
\hline $2 \nu_{50}$ & 485.4 & 485.4 \\
\hline$\nu_{53}+\nu_{47}$ & 505.6 & 489.0 \\
\hline$\nu_{52}+\nu_{48}$ & 503.6 & 494.8 \\
\hline$\nu_{50}+\nu_{49}$ & 517.6 & 499.6 \\
\hline
\end{tabular}

Table D9

Vibrationally Excited States of Leucinol $1, g^{\prime} G g^{\prime} T g$, that Reside under $500 \mathrm{~cm}^{-1}$ in Energy Relative to the Ground State

\begin{tabular}{lrr}
\hline \hline Vibrational Mode & $E^{h} / \mathrm{cm}^{-1}$ & $E^{a} / \mathrm{cm}^{-1}$ \\
\hline$\nu_{63}$ & 48.1 & 12.8 \\
$2 \nu_{63}$ & 96.1 & 19.8 \\
$\nu_{62} \nu_{62}$ & 88.0 & 70.7 \\
$\nu_{63}+\nu_{62}$ & 136.1 & 76.2
\end{tabular}


Table D9

(Continued)

\begin{tabular}{|c|c|c|}
\hline Vibrational Mode & $E^{h} / \mathrm{cm}^{-1}$ & $E^{a} / \mathrm{cm}^{-1}$ \\
\hline$\nu_{61}$ & 135.5 & 128.6 \\
\hline$\nu_{63}+\nu_{61}$ & 183.6 & 137.9 \\
\hline $2 \nu_{62}$ & 176.0 & 139.3 \\
\hline$\nu_{60}$ & 157.0 & 139.4 \\
\hline$\nu_{63}+\nu_{60}$ & 205.1 & 143.8 \\
\hline$\nu_{62}+\nu_{61}$ & 223.5 & 198.0 \\
\hline$\nu_{62}+\nu_{60}$ & 245.0 & 205.8 \\
\hline$\nu_{59}$ & 226.1 & 210.5 \\
\hline$\nu_{63}+\nu_{59}$ & 274.1 & 221.3 \\
\hline$\nu_{58}$ & 246.2 & 229.0 \\
\hline$\nu_{57}$ & 255.9 & 235.7 \\
\hline$\nu_{63}+\nu_{57}$ & 304.0 & 239.5 \\
\hline$\nu_{63}+\nu_{58}$ & 294.3 & 239.8 \\
\hline $2 \nu_{61}$ & 271.1 & 256.9 \\
\hline$\nu_{61}+\nu_{60}$ & 292.6 & 267.8 \\
\hline$\nu_{56}$ & 281.4 & 269.7 \\
\hline $2 \nu_{60}$ & 314.0 & 276.6 \\
\hline$\nu_{63}+\nu_{56}$ & 329.4 & 277.3 \\
\hline$\nu_{62}+\nu_{59}$ & 314.1 & 279.9 \\
\hline$\nu_{62}+\nu_{58}$ & 334.2 & 299.0 \\
\hline$\nu_{62}+\nu_{57}$ & 343.9 & 309.0 \\
\hline$\nu_{55}$ & 315.0 & 313.0 \\
\hline$\nu_{63}+\nu_{55}$ & 363.0 & 325.3 \\
\hline$\nu_{61}+\nu_{59}$ & 361.6 & 339.1 \\
\hline$\nu_{62}+\nu_{56}$ & 369.4 & 341.4 \\
\hline$\nu_{60}+\nu_{59}$ & 383.1 & 349.5 \\
\hline$\nu_{54}$ & 356.5 & 356.4 \\
\hline$\nu_{61}+\nu_{58}$ & 381.8 & 357.0 \\
\hline$\nu_{61}+\nu_{57}$ & 391.4 & 360.2 \\
\hline$\nu_{60}+\nu_{58}$ & 403.2 & 366.4 \\
\hline$\nu_{63}+\nu_{54}$ & 404.6 & 368.0 \\
\hline$\nu_{60}+\nu_{57}$ & 412.9 & 372.3 \\
\hline$\nu_{62}+\nu_{55}$ & 403.0 & 383.0 \\
\hline$\nu_{61}+\nu_{56}$ & 416.9 & 396.5 \\
\hline$\nu_{60}+\nu_{56}$ & 438.4 & 406.9 \\
\hline $2 \nu_{59}$ & 452.1 & 417.7 \\
\hline$\nu_{53}$ & 420.4 & 421.2 \\
\hline$\nu_{52}$ & 426.1 & 421.3 \\
\hline$\nu_{62}+\nu_{54}$ & 444.5 & 426.9 \\
\hline$\nu_{59}+\nu_{58}$ & 472.3 & 430.8 \\
\hline$\nu_{63}+\nu_{52}$ & 474.1 & 433.4 \\
\hline$\nu_{63}+\nu_{53}$ & 468.5 & 434.5 \\
\hline$\nu_{61}+\nu_{55}$ & 450.5 & 441.3 \\
\hline$\nu_{59}+\nu_{57}$ & 482.0 & 442.6 \\
\hline$\nu_{60}+\nu_{55}$ & 472.0 & 451.8 \\
\hline $2 \nu_{58}$ & 492.4 & 455.3 \\
\hline$\nu_{58}+\nu_{57}$ & 502.1 & 460.0 \\
\hline$\nu_{51}$ & 467.0 & 463.2 \\
\hline $2 \nu_{57}$ & 511.8 & 466.4 \\
\hline$\nu_{63}+\nu_{50}$ & 596.5 & 466.4 \\
\hline$\nu_{50}$ & 548.4 & 466.9 \\
\hline$\nu_{63}+\nu_{51}$ & 515.1 & 473.5 \\
\hline$\nu_{59}+\nu_{56}$ & 507.4 & 478.8 \\
\hline$\nu_{61}+\nu_{54}$ & 492.1 & 486.0 \\
\hline$\nu_{62}+\nu_{52}$ & 514.1 & 491.7 \\
\hline$\nu_{62}+\nu_{53}$ & 508.4 & 492.2 \\
\hline$\nu_{60}+\nu_{54}$ & 513.5 & 495.1 \\
\hline$\nu_{58}+\nu_{56}$ & 527.6 & 495.4 \\
\hline
\end{tabular}

Table D10

Vibrationally Excited States of Leucinol 2, $g^{\prime} G g^{\prime} G g^{\prime}$, that Reside under $500 \mathrm{~cm}^{-1}$ in Energy Relative to the Ground State

\begin{tabular}{|c|c|c|}
\hline Vibrational Mode & $E^{h} / \mathrm{cm}^{-1}$ & $E^{a} / \mathrm{cm}^{-1}$ \\
\hline$\nu_{63}$ & 59.2 & 22.2 \\
\hline $2 \nu_{63}$ & 118.3 & 32.7 \\
\hline$\nu_{62}$ & 98.3 & 90.2 \\
\hline$\nu_{63}+\nu_{62}$ & 157.5 & 111.4 \\
\hline$\nu_{61}$ & 131.1 & 123.4 \\
\hline$\nu_{63}+\nu_{61}$ & 190.2 & 143.6 \\
\hline$\nu_{60}$ & 176.6 & 172.5 \\
\hline $2 \nu_{62}$ & 196.6 & 180.5 \\
\hline$\nu_{63}+\nu_{60}$ & 235.8 & 195.0 \\
\hline$\nu_{59}$ & 232.0 & 203.9 \\
\hline$\nu_{62}+\nu_{61}$ & 229.4 & 212.2 \\
\hline$\nu_{63}+\nu_{59}$ & 291.2 & 219.3 \\
\hline$\nu_{58}$ & 248.1 & 221.2 \\
\hline$\nu_{57}$ & 258.6 & 224.2 \\
\hline$\nu_{63}+\nu_{58}$ & 307.2 & 233.7 \\
\hline$\nu_{63}+\nu_{57}$ & 317.7 & 242.9 \\
\hline $2 \nu_{61}$ & 262.1 & 244.8 \\
\hline$\nu_{62}+\nu_{60}$ & 274.9 & 259.3 \\
\hline$\nu_{56}$ & 275.9 & 268.5 \\
\hline$\nu_{63}+\nu_{56}$ & 335.1 & 285.1 \\
\hline$\nu_{62}+\nu_{59}$ & 330.3 & 293.8 \\
\hline$\nu_{61}+\nu_{60}$ & 307.7 & 296.5 \\
\hline$\nu_{62}+\nu_{57}$ & 356.9 & 311.7 \\
\hline$\nu_{62}+\nu_{58}$ & 346.4 & 312.5 \\
\hline$\nu_{55}$ & 321.7 & 313.3 \\
\hline$\nu_{61}+\nu_{59}$ & 363.1 & 326.4 \\
\hline$\nu_{63}+\nu_{55}$ & 380.9 & 333.6 \\
\hline$\nu_{54}$ & 341.8 & 336.6 \\
\hline$\nu_{61}+\nu_{58}$ & 379.1 & 344.2 \\
\hline $2 \nu_{60}$ & 353.2 & 344.2 \\
\hline$\nu_{61}+\nu_{57}$ & 389.6 & 349.2 \\
\hline$\nu_{62}+\nu_{56}$ & 374.2 & 355.0 \\
\hline$\nu_{63}+\nu_{54}$ & 400.9 & 360.0 \\
\hline$\nu_{60}+\nu_{59}$ & 408.6 & 376.3 \\
\hline$\nu_{61}+\nu_{56}$ & 407.0 & 388.6 \\
\hline$\nu_{60}+\nu_{58}$ & 424.7 & 393.5 \\
\hline$\nu_{60}+\nu_{57}$ & 435.2 & 394.7 \\
\hline $2 \nu_{59}$ & 464.0 & 400.4 \\
\hline$\nu_{62}+\nu_{55}$ & 420.0 & 403.3 \\
\hline$\nu_{59}+\nu_{58}$ & 480.1 & 412.9 \\
\hline$\nu_{53}$ & 420.0 & 415.9 \\
\hline$\nu_{52}$ & 435.0 & 423.7 \\
\hline$\nu_{62}+\nu_{54}$ & 440.1 & 426.1 \\
\hline$\nu_{59}+\nu_{57}$ & 490.6 & 426.8 \\
\hline $2 \nu_{57}$ & 517.1 & 427.7 \\
\hline$\nu_{61}+\nu_{55}$ & 452.8 & 436.3 \\
\hline $2 \nu_{58}$ & 496.1 & 436.6 \\
\hline$\nu_{60}+\nu_{56}$ & 452.5 & 436.6 \\
\hline$\nu_{63}+\nu_{53}$ & 479.1 & 437.6 \\
\hline$\nu_{58}+\nu_{57}$ & 506.6 & 443.3 \\
\hline$\nu_{63}+\nu_{52}$ & 494.2 & 443.8 \\
\hline$\nu_{61}+\nu_{54}$ & 472.8 & 460.0 \\
\hline$\nu_{59}+\nu_{56}$ & 507.9 & 464.6 \\
\hline$\nu_{51}$ & 473.2 & 474.4 \\
\hline$\nu_{58}+\nu_{56}$ & 524.0 & 483.2 \\
\hline$\nu_{60}+\nu_{55}$ & 498.3 & 485.7 \\
\hline$\nu_{57}+\nu_{56}$ & 534.5 & 486.7 \\
\hline$\nu_{63}+\nu_{51}$ & 532.4 & 498.1 \\
\hline
\end{tabular}


Table D11

Vibrationally Excited States of Leucinol 3, $g G^{\prime} g T g^{\prime}$, that Reside under $500 \mathrm{~cm}^{-1}$ in Energy Relative to the Ground State

\begin{tabular}{|c|c|c|}
\hline Vibrational Mode & $E^{h} / \mathrm{cm}^{-1}$ & $E^{a} / \mathrm{cm}^{-1}$ \\
\hline$\nu_{63}$ & 48.4 & 10.1 \\
\hline $2 \nu_{63}$ & 96.8 & 13.2 \\
\hline$\nu_{62}$ & 97.6 & 92.8 \\
\hline$\nu_{63}+\nu_{62}$ & 145.9 & 102.2 \\
\hline$\nu_{61}$ & 128.6 & 121.1 \\
\hline$\nu_{63}+\nu_{61}$ & 177.0 & 124.8 \\
\hline$\nu_{60}$ & 176.3 & 163.6 \\
\hline$\nu_{63}+\nu_{60}$ & 224.7 & 172.2 \\
\hline $2 \nu_{62}$ & 195.1 & 186.2 \\
\hline$\nu_{62}+\nu_{61}$ & 226.2 & 214.2 \\
\hline$\nu_{63}+\nu_{56}$ & 323.2 & 215.0 \\
\hline$\nu_{59}$ & 225.4 & 219.7 \\
\hline$\nu_{63}+\nu_{57}$ & 309.4 & 220.4 \\
\hline$\nu_{56}$ & 274.9 & 222.7 \\
\hline$\nu_{57}$ & 261.0 & 223.1 \\
\hline$\nu_{58}$ & 241.6 & 224.1 \\
\hline$\nu_{63}+\nu_{59}$ & 273.7 & 229.9 \\
\hline$\nu_{63}+\nu_{58}$ & 290.0 & 231.3 \\
\hline $2 \nu_{61}$ & 257.2 & 239.9 \\
\hline$\nu_{62}+\nu_{60}$ & 273.9 & 254.8 \\
\hline$\nu_{61}+\nu_{60}$ & 304.9 & 284.0 \\
\hline$\nu_{62}+\nu_{56}$ & 372.4 & 312.3 \\
\hline$\nu_{62}+\nu_{59}$ & 322.9 & 312.5 \\
\hline$\nu_{62}+\nu_{57}$ & 358.6 & 313.5 \\
\hline$\nu_{55}$ & 320.1 & 315.8 \\
\hline$\nu_{62}+\nu_{58}$ & 339.2 & 316.2 \\
\hline$\nu_{63}+\nu_{55}$ & 368.5 & 325.0 \\
\hline $2 \nu_{60}$ & 352.6 & 325.6 \\
\hline$\nu_{61}+\nu_{56}$ & 403.5 & 336.1 \\
\hline$\nu_{61}+\nu_{57}$ & 389.7 & 339.4 \\
\hline$\nu_{61}+\nu_{59}$ & 354.0 & 341.0 \\
\hline$\nu_{61}+\nu_{58}$ & 370.2 & 344.0 \\
\hline$\nu_{54}$ & 355.4 & 350.8 \\
\hline$\nu_{63}+\nu_{54}$ & 403.8 & 362.3 \\
\hline$\nu_{60}+\nu_{59}$ & 401.7 & 382.8 \\
\hline$\nu_{60}+\nu_{56}$ & 451.2 & 384.5 \\
\hline$\nu_{60}+\nu_{57}$ & 437.3 & 385.5 \\
\hline$\nu_{60}+\nu_{58}$ & 417.9 & 387.2 \\
\hline$\nu_{53}$ & 405.9 & 407.4 \\
\hline$\nu_{62}+\nu_{55}$ & 417.7 & 407.6 \\
\hline$\nu_{63}+\nu_{53}$ & 454.3 & 417.9 \\
\hline$\nu_{57}+\nu_{56}$ & 535.9 & 427.3 \\
\hline $2 \nu_{56}$ & 549.7 & 433.2 \\
\hline $2 \nu_{57}$ & 522.1 & 437.3 \\
\hline$\nu_{61}+\nu_{55}$ & 448.7 & 437.9 \\
\hline$\nu_{59}+\nu_{57}$ & 486.4 & 437.9 \\
\hline$\nu_{52}$ & 440.1 & 439.5 \\
\hline$\nu_{59}+\nu_{58}$ & 467.0 & 439.7 \\
\hline$\nu_{58}+\nu_{56}$ & 450.7 & 439.8 \\
\hline$\nu_{58}+\nu_{57}$ & 500.2 & 440.1 \\
\hline$\nu_{62}+\nu_{54}$ & 516.5 & 442.3 \\
\hline $2 \nu_{58}$ & 502.6 & 442.9 \\
\hline$\nu_{63}+\nu_{52}$ & 453.0 & 443.0 \\
\hline$\nu_{63}+\nu_{50}$ & 483.2 & 446.7 \\
\hline$\nu_{50}$ & 488.5 & 450.7 \\
\hline$\nu_{51}$ & 579.0 & 451.3 \\
\hline$\nu_{63}+\nu_{51}$ & 530.6 & 451.5 \\
\hline$\nu_{61}+\nu_{54}$ & 477.5 & 454.5 \\
\hline$\nu_{60}+\nu_{55}$ & 525.9 & 459.3 \\
\hline$\nu_{62}+\nu_{53}$ & 484.0 & 473.6 \\
\hline$\nu_{60}+\nu_{54}$ & 496.4 & 480.3 \\
\hline$\nu_{61}+\nu_{53}$ & 503.5 & 500.3 \\
\hline
\end{tabular}


Appendix E

Partition Functions for Assigned Species

This appendix contains the partition functions for the assigned species in this work. For those species whose fits encompass data across both the $2-26 \mathrm{GHz}$ and $75-110 \mathrm{GHz}$ ranges and, for completeness, the ground-state conformers of leucinol, two sets of rotational partition functions are tabulated; one set corresponds to the $2 I+1=1$ case (without hyperfine splitting), and one set corresponds to the $2 I+1=3$ case (with hyperfine splitting). The values were all calculated using the SPCAT program (Pickett 1991), which performs a direct

Table E1

Rotational Partition Functions $\left(Q_{\text {rot }}\right.$ ) for the Isotopologues of Alaninol 1, $g^{\prime} G g^{\prime}$, where $J_{\max }=220$ and $K_{\max }=40$

\begin{tabular}{|c|c|c|c|c|c|}
\hline$T / K$ & $\begin{array}{l}Q_{\text {rot }}{ }^{13} \mathrm{C}(1) \\
{[2 I+1=3]}\end{array}$ & $\begin{array}{c}Q_{\text {rot }}{ }^{13} \mathrm{C}(2) \\
{[2 I+1=3]}\end{array}$ & $\begin{array}{c}Q_{\text {rot }}{ }^{13} \mathrm{C}(3) \\
{[2 I+1=3]}\end{array}$ & $\begin{array}{c}Q_{\mathrm{rot}}{ }^{15} \mathrm{~N} \\
{[2 I+1=1]}\end{array}$ & $\begin{array}{c}Q_{\text {rot }}{ }^{18} \mathrm{O} \\
{[2 I+1=3]}\end{array}$ \\
\hline 300 & 290093.6 & 283781.0 & 285406.4 & 96069.4 & 140294.9 \\
\hline 225 & 192917.2 & 188669.5 & 189815.0 & 63955.2 & 115642.5 \\
\hline 150 & 106568.5 & 104197.5 & 104862.5 & 35363.4 & 81707.9 \\
\hline 75 & 37822.0 & 36977.3 & 37218.1 & 12556.1 & 36574.6 \\
\hline 37.5 & 13377.2 & 13078.6 & 13163.8 & 4441.0 & 13606.7 \\
\hline 18.75 & 4734.4 & 4628.8 & 4658.9 & 1571.8 & 4826.6 \\
\hline 9.375 & 1677.6 & 1640.3 & 1650.9 & 557.0 & 1710.2 \\
\hline 1.5 & 110.0 & 107.6 & 108.3 & 36.5 & 112.1 \\
\hline
\end{tabular}

Table E2

Rotational $\left(Q_{\mathrm{rot}}\right)$ and Vibrational $\left(Q_{\mathrm{vib}}\right)$ Partition Functions for Alaninol 2, $g G^{\prime} g$, where $J_{\max }=220$ and $K_{\max }=40$

\begin{tabular}{|c|c|c|c|c|c|c|c|}
\hline$T / K$ & $\begin{array}{l}Q_{\text {rot }} \text { Parent } \\
{[2 I+1=1]}\end{array}$ & $\begin{array}{l}Q_{\text {rot }} \text { Parent } \\
{[2 I+1=3]}\end{array}$ & $Q_{\mathrm{vib}}$ & $\begin{array}{l}Q_{\text {rot }}{ }^{13} \mathrm{C}(1) \\
{[2 I+1=3]}\end{array}$ & $\begin{array}{c}Q_{\text {rot }}{ }^{13} \mathrm{C}(2) \\
{[2 I+1=3]}\end{array}$ & $\begin{array}{c}Q_{\text {rot }}{ }^{13} \mathrm{C}(3) \\
{[2 I+1=3]}\end{array}$ & $\begin{array}{c}Q_{\text {rot }}{ }^{15} \mathrm{~N} \\
{[2 I+1=1]}\end{array}$ \\
\hline 300 & 86814.4 & 260443.1 & 4.082 & 265983.8 & 261659.2 & 263141.7 & 88464.1 \\
\hline 225 & 58300.5 & 174901.5 & 2.932 & 178787.4 & 175745.7 & 176770.5 & 59483.1 \\
\hline 150 & 32518.4 & 97555.1 & 1.829 & 99821.3 & 98042.4 & 98631.4 & 33222.8 \\
\hline 75 & 11597.7 & 34793.1 & 1.111 & 35622.2 & 34970.3 & 35184.0 & 11858.5 \\
\hline 37.5 & 4102.7 & 12308.0 & 1.005 & 12601.4 & 12370.7 & 12446.3 & 4195.0 \\
\hline 18.75 & 1452.2 & 4356.6 & 1.000 & 4460.3 & 4378.8 & 4405.5 & 1484.9 \\
\hline 9.375 & 514.8 & 1544.3 & 1.000 & 1581.0 & 1552.1 & 1561.6 & 526.3 \\
\hline 1.5 & 33.9 & 101.6 & 1.000 & 104.0 & 102.2 & 102.8 & 34.6 \\
\hline
\end{tabular}

Table E3

Rotational $\left(Q_{\text {rot }}\right)$ and Vibrational $\left(Q_{\text {vib }}\right)$ Partition Functions for Valinol $1, g^{\prime} G g^{\prime} G^{\prime}$, where $J_{\max }=310$ and $K_{\max }=40$

\begin{tabular}{|c|c|c|c|c|c|c|c|c|c|c|}
\hline$T / K$ & $\begin{array}{l}Q_{\text {rot }} \text { Parent } \\
{[2 I+1=1]}\end{array}$ & $\begin{array}{l}Q_{\text {rot }} \text { Parent } \\
{[2 I+1=3]}\end{array}$ & $Q_{\text {vib }}$ & $\begin{array}{c}Q_{\text {rot }}{ }^{13} \mathrm{C}(1) \\
{[2 I+1=3]}\end{array}$ & $\begin{array}{c}Q_{\text {rot }}{ }^{13} \mathrm{C}(2) \\
{[2 I+1=3]}\end{array}$ & $\begin{array}{l}Q_{\mathrm{rot}}{ }^{13} \mathrm{C}(3) \\
{[2 I+1=3]}\end{array}$ & $\begin{array}{c}Q_{\text {rot }}{ }^{13} \mathrm{C}(4) \\
{[2 I+1=3]}\end{array}$ & $\begin{array}{c}Q_{\text {rot }}{ }^{13} \mathrm{C}(5) \\
{[2 I+1=3]}\end{array}$ & $\begin{array}{c}Q_{\mathrm{rot}}{ }^{15} \mathrm{~N} \\
{[2 I+1=1]}\end{array}$ & $\begin{array}{c}Q_{\text {rot }}{ }^{18} \mathrm{O} \\
{[2 I+1=3]}\end{array}$ \\
\hline 300 & 252432.8 & 757298.3 & 9.914 & 760706.5 & 757585.8 & 762542.6 & 769097.7 & 770495.6 & 254852.1 & 787185.8 \\
\hline 225 & 174088.4 & 522265.2 & 6.818 & 524635.4 & 522481.6 & 526017.3 & 530950.9 & 531533.2 & 175945.2 & 542940.1 \\
\hline 150 & 100345.1 & 301035.2 & 3.767 & 302418.1 & 301175.4 & 303307.7 & 306485.0 & 306512.5 & 101567.5 & 313005.2 \\
\hline 75 & 36815.1 & 110445.2 & 1.579 & 110960.6 & 110504.1 & 111325.3 & 112629.6 & 112511.5 & 37327.1 & 114859.6 \\
\hline 37.5 & 13065.4 & 39196.2 & 1.087 & 39380.2 & 39218.2 & 39512.2 & 39984.8 & 39933.8 & 13251.7 & 40764.5 \\
\hline 18.75 & 4621.7 & 13865.1 & 1.005 & 13930.4 & 13873.1 & 13977.1 & 14144.2 & 14126.1 & 4687.7 & 14419.8 \\
\hline 9.375 & 1635.8 & 4907.3 & 1.000 & 4930.3 & 4910.1 & 4946.9 & 5006.0 & 4999.6 & 1659.1 & 5103.4 \\
\hline 1.5 & 105.9 & 317.7 & 1.000 & 319.2 & 317.9 & 320.2 & 324.0 & 323.6 & 107.4 & 330.3 \\
\hline
\end{tabular}

Table E4

Rotational $\left(Q_{\text {rot }}\right)$ and Vibrational $\left(Q_{\text {vib }}\right)$ Partition Functions for Valinol 2, $g^{\prime} G g^{\prime} G$, where $J_{\max }=310$ and $K_{\max }=40$

\begin{tabular}{|c|c|c|c|c|c|c|c|c|c|}
\hline$T / K$ & $\begin{array}{l}Q_{\text {rot }} \text { Parent } \\
{[2 I+1=1]}\end{array}$ & $\begin{array}{l}Q_{\text {rot }} \text { Parent } \\
{[2 I+1=3]}\end{array}$ & $Q_{\text {vib }}$ & $\begin{array}{c}Q_{\mathrm{rot}}{ }^{13} \mathrm{C}(1) \\
{[2 I+1=3]}\end{array}$ & $\begin{array}{c}Q_{\text {rot }}{ }^{13} \mathrm{C}(2) \\
{[2 I+1=3]}\end{array}$ & $\begin{array}{c}Q_{\text {rot }}{ }^{13} \mathrm{C}(3) \\
{[2 I+1=3]}\end{array}$ & $\begin{array}{c}Q_{\text {rot }}{ }^{13} \mathrm{C}(4) \\
{[2 I+1=3]}\end{array}$ & $\begin{array}{l}Q_{\mathrm{rot}}{ }^{13} \mathrm{C}(5) \\
{[2 I+1=3]}\end{array}$ & $\begin{array}{c}Q_{\text {rot }}{ }^{15} \mathrm{~N} \\
{[2 I+1=1}\end{array}$ \\
\hline 300 & 249015.5 & 747046.6 & 9.296 & 752640.8 & 747579.4 & 750680.5 & 760570.8 & 758019.6 & 251165.8 \\
\hline 225 & 171206.0 & 513617.9 & 6.264 & 517596.2 & 514023.9 & 516176.2 & 523141.5 & 521654.5 & 172858.2 \\
\hline 150 & 98274.2 & 294822.6 & 3.359 & 297209.9 & 295086.7 & 296338.2 & 300466.7 & 299822.8 & 99359.2 \\
\hline 75 & 35893.5 & 107680.5 & 1.418 & 108592.4 & 107788.9 & 108252.1 & 109810.5 & 109657.5 & 36343.0 \\
\hline 37.5 & 12727.4 & 38182.2 & 1.046 & 38507.9 & 38221.3 & 38385.9 & 38941.4 & 38892.3 & 12890.0 \\
\hline 18.75 & 4502.0 & 13506.1 & 1.001 & 13621.3 & 13520.0 & 13578.1 & 13774.5 & 13757.2 & 4559.6 \\
\hline 9.375 & 1593.5 & 4780.4 & 1.000 & 4821.1 & 4785.3 & 4805.8 & 4875.3 & 4869.2 & 1613.8 \\
\hline 1.5 & 103.2 & 309.6 & 1.000 & 312.3 & 310.0 & 311.3 & 315.8 & 315.4 & 104.5 \\
\hline
\end{tabular}


Table E5

Rotational $\left(Q_{\text {rot }}\right)$ and Vibrational $\left(Q_{\text {vib }}\right)$ Partition Functions for Valinol 3, $g^{\prime} G g^{\prime} T$, where $J_{\max }=310$ and $K_{\max }=40$

\begin{tabular}{lccc}
\hline \hline$T / K$ & $Q_{\text {rot }}$ Parent & $Q_{\text {rot }}$ Parent \\
{$[2 I+1=1]$} & $Q_{\text {vib }}$ \\
& {$[2 I=3]$} & 729086.6 & 505561.1 \\
200 & 243028.9 & 293700.8 & 7.490 \\
225 & 168520.4 & 108760.9 & 4.377 \\
150 & 97900.3 & 38676.7 & 1.987 \\
75 & 36253.6 & 13682.5 & 1.263 \\
37.5 & 12892.2 & 4843.0 & 1.039 \\
18.75 & 4560.8 & 313.8 & 1.001 \\
9.375 & 1614.3 & 1.000 \\
1.5 & 104.6 & & \\
\hline
\end{tabular}

Table E6

Rotational $\left(Q_{\mathrm{rot}}\right)$ and Vibrational $\left(Q_{\mathrm{vib}}\right)$ Partition Functions for Valinol $4, g G^{\prime} g G^{\prime}$, where $J_{\max }=310$ and $K_{\max }=40$

\begin{tabular}{lrrr}
\hline \hline$T / K$ & $Q_{\text {rot }}$ Parent & $Q_{\text {rot }}$ Parent & $Q_{\text {vib }}$ \\
& {$[2 I+1=1]$} & $67+1=3]$ & 9.614 \\
300 & 224996.6 & 469630.0 & 6.683 \\
225 & 156543.3 & 274175.7 & 3.819 \\
150 & 91391.9 & 102169.2 & 1.721 \\
75 & 34056.4 & 36391.0 & 1.152 \\
37.5 & 12130.3 & 12874.6 & 1.015 \\
18.75 & 4291.5 & 4557.2 & 1.000 \\
9.375 & 1519.1 & 295.5 & 1.000 \\
1.5 & 98.5 & & \\
\hline
\end{tabular}

Table E7

Rotational $\left(Q_{\text {rot }}\right)$ and Vibrational $\left(Q_{\text {vib }}\right)$ Partition Functions for Leucinol $1, g^{\prime} G g^{\prime} T g$, where $J_{\max }=400$ and $K_{\max }=40$

\begin{tabular}{|c|c|c|c|c|c|c|c|c|c|c|}
\hline$T / K$ & $\begin{array}{l}Q_{\text {rot }} \text { Parent } \\
{[2 I+1=1]}\end{array}$ & $\begin{array}{l}Q_{\text {rot }} \text { Parent } \\
{[2 I+1=3]}\end{array}$ & $Q_{\text {vib }}$ & $\begin{array}{c}Q_{\text {rot }}{ }^{13} \mathrm{C}(1) \\
{[2 I+1=3]}\end{array}$ & $\begin{array}{c}Q_{\text {rot }}{ }^{13} \mathrm{C}(2) \\
{[2 I+1=3]}\end{array}$ & $\begin{array}{l}Q_{\text {rot }}{ }^{13} \mathrm{C}(3) \\
{[2 I+1=3]}\end{array}$ & $\begin{array}{l}Q_{\text {rot }}{ }^{13} \mathrm{C}(4) \\
{[2 I+1=3]}\end{array}$ & $\begin{array}{c}Q_{\text {rot }}{ }^{13} \mathrm{C}(5) \\
{[2 I+1=3]}\end{array}$ & $\begin{array}{l}Q_{\text {rot }}{ }^{13} \mathrm{C}(6) \\
{[2 I+1=3]}\end{array}$ & $\begin{array}{c}Q_{\mathrm{rot}}{ }^{15} \mathrm{~N} \\
{[2 I+1=1]}\end{array}$ \\
\hline 300 & 418643.6 & 1255930.9 & 16.788 & 1267098.9 & 1257102.9 & 1258150.5 & 1263217.0 & 1278750.7 & 1272943.8 & 422080.7 \\
\hline 225 & 288565.0 & 865694.9 & 12.076 & 873649.0 & 866529.4 & 867406.3 & 870728.9 & 881736.0 & 8781863 & 291206.6 \\
\hline 150 & 166217.5 & 498652.4 & 7.295 & 503441.4 & 499149.9 & 499783.4 & 501561.4 & 508140.1 & 506463.9 & 167958.0 \\
\hline 75 & 60934.3 & 182802.8 & 3.488 & 184643.8 & 182991.7 & 183277.2 & 183873.2 & 186381.0 & 185920.3 & 61662.6 \\
\hline 37.5 & 21618.2 & 64854.6 & 2.228 & 65513.6 & 64922.6 & 65027.4 & 65234.7 & 66130.5 & 65978.2 & 21883.0 \\
\hline 18.75 & 7645.3 & 22935.8 & 1.602 & 23168.8 & 22960.0 & 22997.0 & 23070.2 & 23386.8 & 23333.2 & 7739.0 \\
\hline 9.375 & 2704.8 & 8114.3 & 1.189 & 8196.7 & 8122.9 & 8136.0 & 8161.8 & 8273.8 & 8254.9 & 2737.9 \\
\hline 1.5 & 174.4 & 523.2 & 1.000 & 528.5 & 523.8 & 524.6 & 526.3 & 533.4 & 532.2 & 176.5 \\
\hline
\end{tabular}

Table E8

Rotational $\left(Q_{\text {rot }}\right)$ and Vibrational $\left(Q_{\text {vib }}\right)$ Partition Functions for Leucinol $2, g^{\prime} G g^{\prime} G g^{\prime}$, where $J_{\max }=400$ and $K_{\max }=40$

\begin{tabular}{lcrr}
\hline \hline$T / K$ & $\begin{array}{l}Q_{\text {rot }} \text { Parent } \\
{[2 I+1=1]}\end{array}$ & $\begin{array}{c}Q_{\text {rot }} \text { Parent } \\
{[2 I+1=3]}\end{array}$ \\
\hline 300 & 390664.9 & 1171994.6 & $Q_{\text {vib }}$ \\
225 & 271684.2 & 815052.7 & 15.663 \\
150 & 158505.8 & 475517.5 & 11.126 \\
75 & 59012.5 & 177037.4 & 6.502 \\
37.5 & 21012.1 & 63036.3 & 2.889 \\
18.75 & 7432.0 & 22296.0 & 1.774 \\
9.375 & 2629.5 & 7888.5 & 1.265 \\
1.5 & 169.7 & 509.0 & 1.040 \\
\end{tabular}


Table E9

Rotational $\left(Q_{\text {rot }}\right)$ and Vibrational $\left(Q_{\text {vib }}\right)$ Partition Functions for Leucinol 3, $g G^{\prime} g T g^{\prime}$, where $J_{\max }=400$ and $K_{\max }=40$

\begin{tabular}{lrrr}
\hline \hline$T / K$ & $\begin{array}{c}Q_{\text {rot }} \text { Parent } \\
{[2 I+1=1]}\end{array}$ & $\begin{array}{c}Q_{\text {rot }} \text { Parent } \\
{[2 I+1=3]}\end{array}$ \\
\hline 300 & 404475.5 & 1213426.5 & $Q_{\text {vib }}$ \\
225 & 281720.5 & 845161.4 & 16.683 \\
150 & 164738.9 & 494216.5 & 11.902 \\
75 & 61519.6 & 184558.9 & 7.071 \\
37.5 & 21923.0 & 65769.0 & 3.386 \\
18.75 & 7754.2 & 23262.7 & 2.353 \\
9.375 & 2743.4 & 8230.3 & 1.825 \\
1.5 & 177.0 & 530.9 & 1.344 \\
\hline
\end{tabular}

summation over the rotational energy levels (Kisiel 2015; Endres et al. 2016). The temperatures are the standard temperatures implemented in the JPL database (Pickett et al. 1998) and SPCAT (Pickett 1991). The values of $J_{\max }$ and $K_{\max }$ considered in the summation calculations are detailed in the relevant table captions. Vibrational partition function contributions are listed alongside all ground-state conformers. These values were determined by considering the anharmonic energies of the vibrational states up to $500 \mathrm{~cm}^{-1}$ with respect to the ground-state energy and are all given to three decimal places.

\section{Appendix F}

\section{Machine-readable Tables for Assignments and Predictions}

Machine-readable tables available online contain the experimentally measured transitions extracted from the .fit files produced by the SPFIT program. Note that, where global fits combining low- and high-frequency data were performed, the transitions have quantum number labeling of the form $J K_{a} K_{c}$ $F \leftarrow J^{\prime} K_{a}{ }^{\prime} K_{c}{ }^{\prime} F^{\prime}$. The lines in the $W$-band do not exhibit hyperfine splitting, making the $F$ quantum number redundant. In these cases, $F$ is set to the same value as $J$. An example of the machine-readable table is shown in Table F1. There are 49 total tables available in the .tar.gz package associated with Table F1.

Table F2 is a representative of the predicted spectra with hyperfine splitting up to $50 \mathrm{GHz}$ at $1.5 \mathrm{~K}$. The .tar.gz package associated with Table F2 contains 76 machine-readable tables. The first 38 provide the predicted spectra with hyperfine splitting up to $50 \mathrm{GHz}$ at $1.5 \mathrm{~K}$. The remaining files have hyperfine splitting up to $50 \mathrm{GHz}$ at $9.375 \mathrm{~K}$. These machinereadable tables take the format shown in the sample of Table F2.

For species that have been assigned in the $W$-band region, 20 machine-readable tables are available in the tar.gz package associated with Table F3. These files contain the predicted spectra without hyperfine splitting up to $300 \mathrm{GHz}$ at $300 \mathrm{~K}$, and all of these take the form of the example in Table F3. All predictions were produced by using the converged $Q_{\text {rot }}$ values (tabulated in Appendix E) at the specified temperatures.

The $J_{\max }$ values for each prediction are as follows: alaninol $J_{\max }=12$ for the predictions up to $50 \mathrm{GHz}$ and $J_{\max }=55$ for the predictions up to $300 \mathrm{GHz}$; valinol $J_{\max }=20$ for the predictions up to $50 \mathrm{GHz}$ and $J_{\max }=115$ for the predictions up to $300 \mathrm{GHz}$; leucinol $J_{\max }=30$ for the predictions up to $50 \mathrm{GHz}$ and $J_{\max }=170$ for the predictions up to $300 \mathrm{GHz}$. 
Table F1

A Sample of the Table Containing the Experimentally Measured Transitions for Alaninol $1, g^{\prime} G g^{\prime}$

\begin{tabular}{ccccccccccc}
\hline \hline$J$ & $K_{a}$ & $K_{c}$ & $F$ & $J^{\prime}$ & $K_{a}{ }^{\prime}$ & $K_{c}{ }^{\prime}$ & $F^{\prime}$ & Observed/MHz & Observed - Calculated $/ \mathrm{MHz}$ & Blend $/ \mathrm{MHz}$ \\
\hline 3 & 1 & 3 & 3 & 3 & 0 & 3 & 2 & 2610.3327 & 0.0020 & -0.0024 \\
3 & 1 & 3 & 3 & 3 & 0 & 3 & 4 & 2610.7466 & 0.0028 & $\ldots$ \\
3 & 1 & 3 & 2 & 3 & 0 & 3 & 2 & 2611.5936 & $\ldots$ & $\ldots$ \\
3 & 1 & 3 & 4 & 3 & 0 & 3 & 4 & 2611.6835 & $\ldots$ & $\ldots$ \\
3 & 1 & 3 & 3 & 3 & 0 & 3 & 3 & 2611.9457 & $\ldots$ & $\ldots$ \\
\hline
\end{tabular}

(This table is available in its entirety in machine-readable form.)

Table F2

A Sample of the Table Containing the SPCAT Prediction for Alaninol $1, g^{\prime} G^{\prime}$, at $1.5 \mathrm{~K}$ up to $50 \mathrm{GHz}$

\begin{tabular}{lccccccccccccccc}
\hline \hline $\begin{array}{c}\text { Frequency } \\
(\mathrm{MHz})\end{array}$ & $\begin{array}{c}\text { Uncertainty } \\
(\mathrm{MHz})\end{array}$ & $\begin{array}{c}\text { log[intensity] } \\
\left.(\mathrm{nm})^{* *} 2 . \mathrm{MHz}\right)\end{array}$ & dof & $\begin{array}{c}\text { E0 } \\
\left(\mathrm{cm}^{* *}-1\right)\end{array}$ & gup & MolTag & Code & $J$ & $K_{a}$ & $K_{c}$ & $F$ & $J^{\prime}$ & $K_{a}{ }^{\prime}$ & $K_{c}{ }^{\prime}$ & $F^{\prime}$ \\
\hline 7.2332 & 0.0000 & -9.8823 & 3 & 5.7462 & 11 & 91 & 304 & 5 & 4 & 1 & 5 & 5 & 4 & 2 & 5 \\
7.2337 & 0.0000 & -9.7924 & 3 & 5.7462 & 13 & 91 & 304 & 5 & 4 & 1 & 6 & 5 & 4 & 2 & 6 \\
7.2338 & 0.0000 & -9.9576 & 3 & 5.7462 & 9 & 91 & 304 & 5 & 4 & 1 & 4 & 5 & 4 & 2 & 4 \\
8.9937 & 0.0037 & -9.5450 & 3 & 2.7083 & 5 & 91 & 304 & 3 & 3 & 0 & 2 & 3 & 3 & 1 & 3 \\
9.5278 & 0.0027 & -9.4896 & 3 & 2.7083 & 9 & 91 & 304 & 3 & 3 & 0 & 4 & 3 & 3 & 1 & 3 \\
\hline
\end{tabular}

(This table is available in its entirety in machine-readable form.)

Table F3

A Sample of the Table Containing the SPCAT Prediction for Alaninol $1, g^{\prime} G^{\prime}$, at $300 \mathrm{~K}$ up to $300 \mathrm{GHz}$

\begin{tabular}{lccccccccccccc}
\hline \hline Frequency $(\mathrm{MHz})$ & Uncertainty $(\mathrm{MHz})$ & $\log \left[\right.$ intensity $\left(\mathrm{nm}^{* *} 2 . \mathrm{MHz}\right)$ & dof & E0 $\left(\mathrm{cm}^{* *}-1\right)$ & gup & MolTag & Code & $J$ & $K_{a}$ & $K_{c}$ & $J^{\prime}$ & $K_{a}{ }^{\prime}$ & $K_{c}{ }^{\prime}$ \\
\hline 223.3978 & 0.0072 & -9.8538 & 3 & 72.4782 & 47 & 91 & 303 & 23 & 9 & 14 & 23 & 9 & 15 \\
233.5689 & 0.0196 & -9.8869 & 3 & 144.2424 & 67 & 91 & 303 & 33 & 12 & 21 & 33 & 12 & 22 \\
268.7516 & 0.0058 & -9.6970 & 3 & 55.5031 & 41 & 91 & 303 & 20 & 8 & 12 & 20 & 8 & 13 \\
273.5783 & 0.0379 & -9.8454 & 3 & 208.7014 & 81 & 91 & 303 & 40 & 14 & 26 & 40 & 14 & 27 \\
296.4338 & 0.0187 & -9.6619 & 3 & 119.8068 & 61 & 91 & 303 & 30 & 11 & 19 & 30 & 11 & 20 \\
\hline
\end{tabular}

(This table is available in its entirety in machine-readable form.)

\section{Appendix G}

\section{Comparing Amino Alcohols with iso-Propyl Cyanide}

The figures in this appendix compare the predictions of the lowest energy conformers of alaninol, valinol, and leucinol to that of IPC at $300 \mathrm{~K}$ in the $75-110 \mathrm{GHz}$ region. The discussion can be found in the main text.
Figures G1-G3 show individual comparisons between IPC and the respective amino alcohols in this study. Figure G4 shows a zoom-in of the predictions of all three amino alcohols and IPC around 88,250 $\mathrm{MHz}$. 


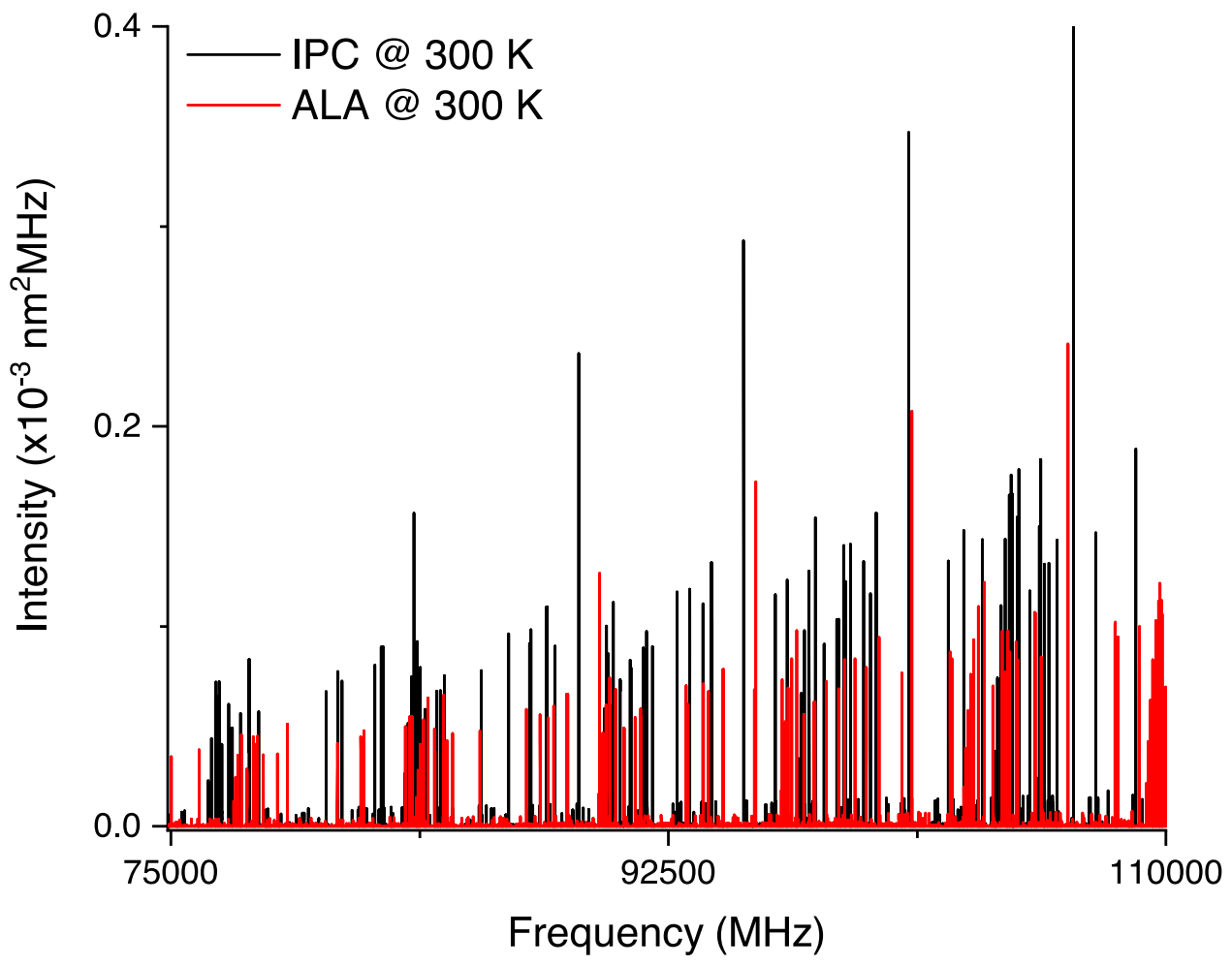

Figure G1. A comparison of the distribution of rotational transitions for iso-propyl cyanide (IPC) and alaninol $1, g^{\prime} G g^{\prime}$, at $300 \mathrm{~K}$ in the $75-110 \mathrm{GHz}$ frequency region. The similarity in the number and intensity distribution shows that alaninol is an attractive target for interstellar searches in areas where, for example, IPC may be found.

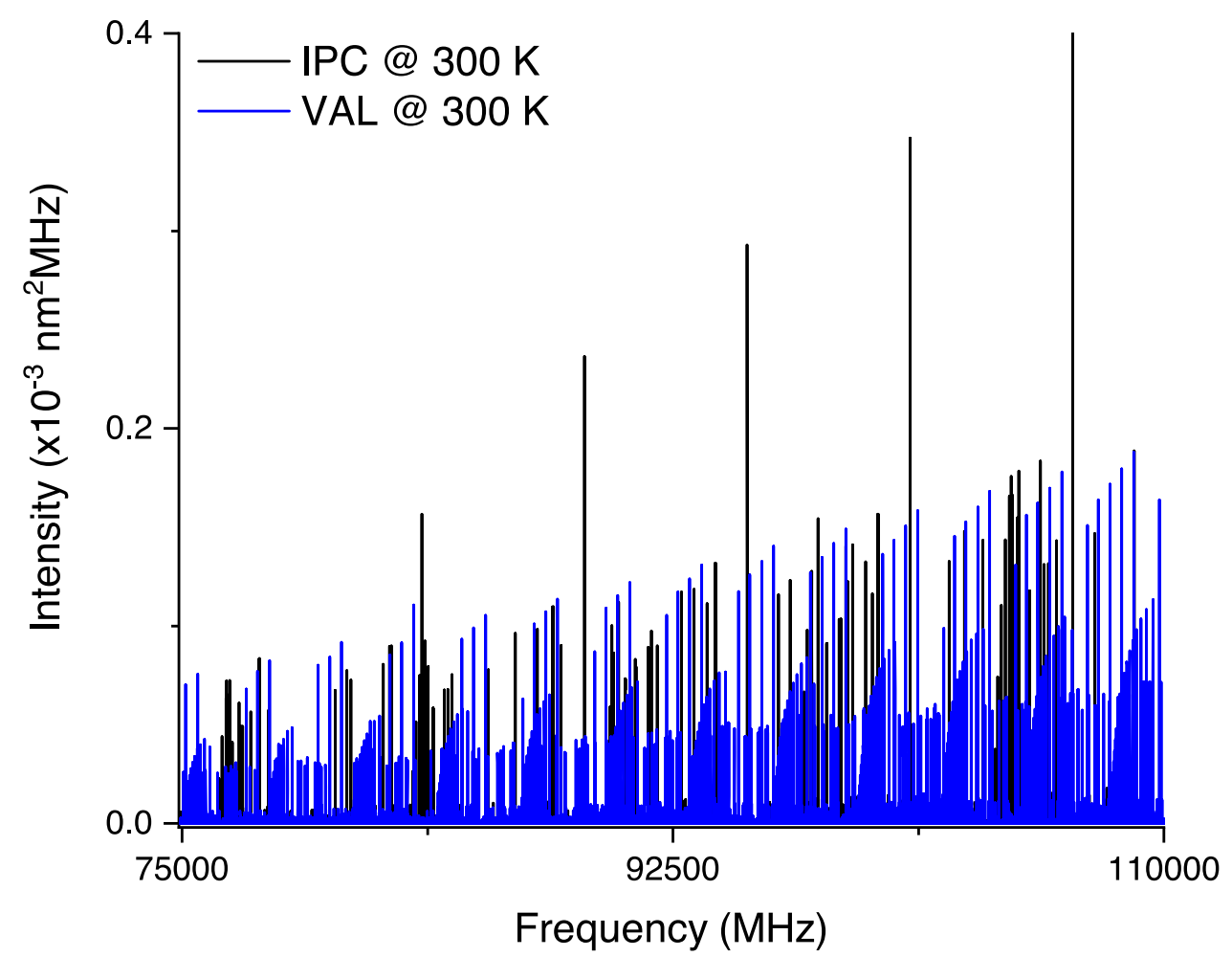

Figure G2. A comparison of the distribution of rotational transitions for iso-propyl cyanide (IPC) and valinol $1, g^{\prime} G g^{\prime} G^{\prime}$, at $300 \mathrm{~K}$ in the $75-110 \mathrm{GHz}$ frequency region. The number of rotational transitions available is larger than those available for IPC, and the intensities for the majority of the transitions are of the same order of magnitude. 


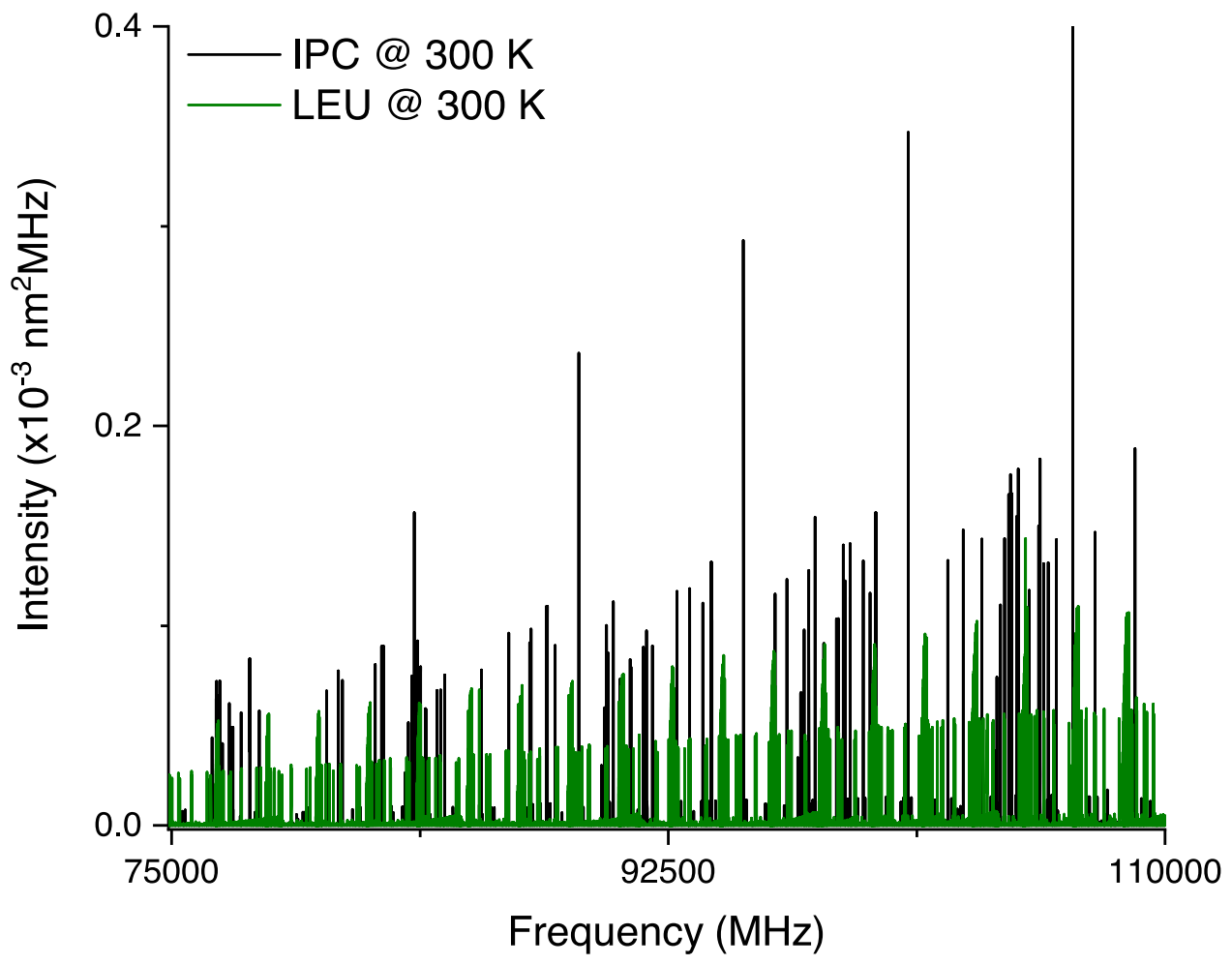

Figure G3. A comparison of the distribution of rotational transitions for iso-propyl cyanide (IPC) and leucinol $1, g^{\prime} G g^{\prime} T g$, at $300 \mathrm{~K}$ in the $75-110 \mathrm{GHz}$ frequency region. The more regularly repeating pattern for leucinol shows a greater number of lines in the $W$-band region when compared to alaninol and valinol, but the intensities are slightly lower.

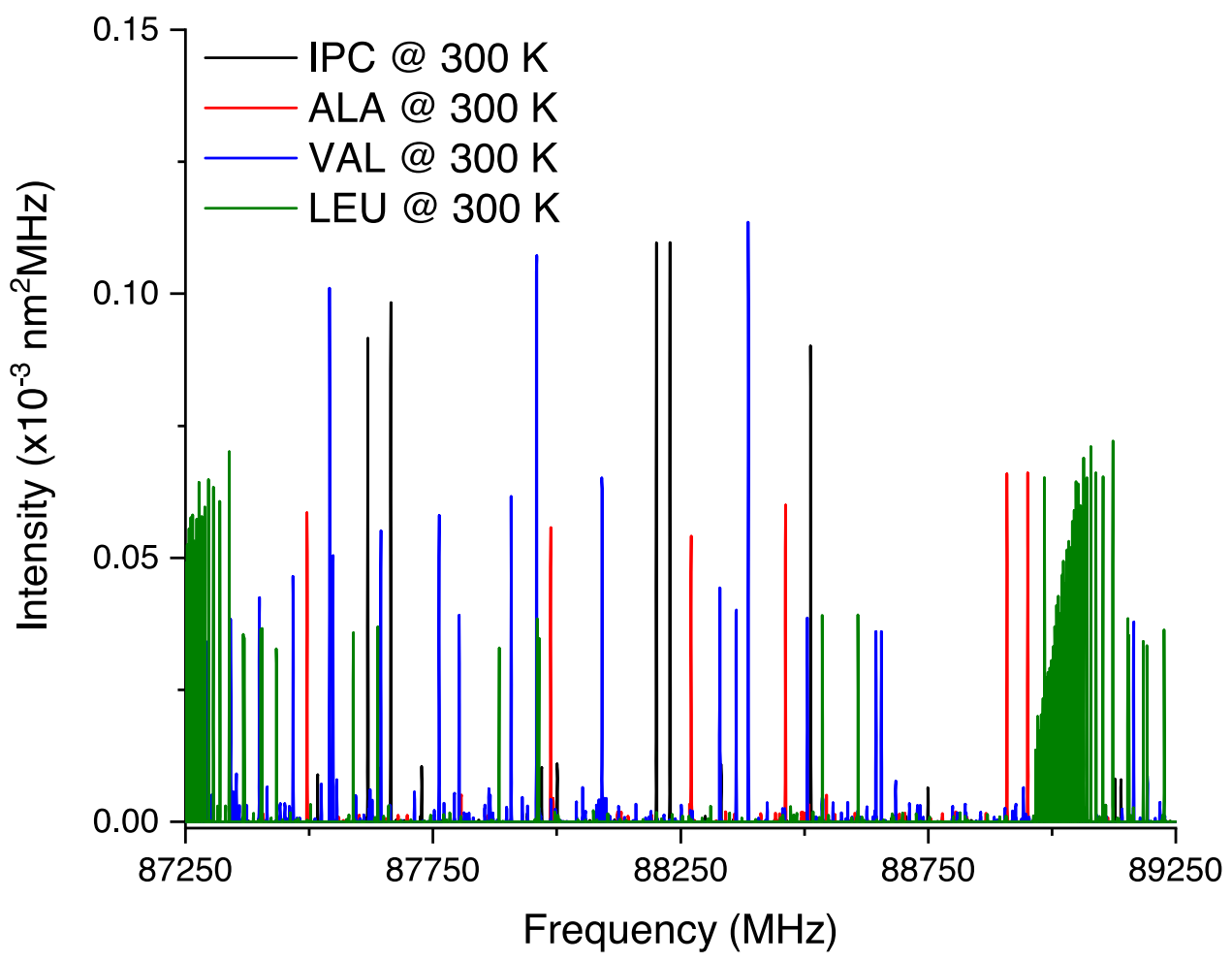

Figure G4. A comparison of the distribution of rotational transitions for iso-propyl cyanide (IPC) and the lowest energy conformers alaninol $1, g^{\prime} G g^{\prime}$, valinol 1 , $g^{\prime} G g^{\prime} G^{\prime}$, and leucinol $1, g^{\prime} G g^{\prime} T g$, at $300 \mathrm{~K}$ around 88,250 MHz. The zoom-in shows the distinct and similarly intense lines for the amino alcohols and IPC. 


\section{ORCID iDs}

Benjamin E. Arenas (1) https://orcid.org/0000-0001-

9471-778X

Mariyam Fatima (10) https://orcid.org/0000-0002-2884-8260

Cristóbal Pérez (i) https://orcid.org/0000-0001-5248-5212

Amanda L. Steber (1) https://orcid.org/0000-0002-8203-2174

Melanie Schnell 1 i https://orcid.org/0000-0001-7801-7134

\section{References}

Altwegg, K., Balsiger, H., Bar-Nun, A., et al. 2016, SciA, 2, e1600285

Arenas, B. E., Gruet, S., Steber, A. L., Giuliano, B. M., \& Schnell, M. 2017, PCCP, 19, 1751

Blanco, S., Lesarri, A., López, J. C., \& Alonso, J. L. 2004, JAChS, 126, 11675

Bogey, M., Dubus, H., \& Guillemin, J. 1990, JMoSp, 143, 180

Cocinero, E. J., Lesarri, A., Grabow, J. U., López, J. C., \& Alonso, J. L. 2007, ChemPhysChem, 8, 599

Cunningham, M. R., Jones, P. A., Godfrey, P. D., et al. 2007, MNRAS, 376, 1201

Domingos, S. R., Pérez, C., Medcraft, C., Pinacho, P., \& Schnell, M. 2016, PCCP, 18, 16682

Durig, J., \& Li, Y. 1974, JMoSt, 21, 289

Ellingsen, B. H., Marstokk, K. M., \& Møllendal, H. 1978, JMoSt, 48, 9

Endres, C. P., Schlemmer, S., Schilke, P., Stutzki, J., \& Müller, H. S. 2016, JMoSp, 327, 95

Fatima, M., Pérez, C., Arenas, B. E., Schnell, M., \& Steber, A. L. 2020, PCCP, 22, 17042

Fausto, R., Cacela, C., \& Duarte, M. L. 2000, JMoSt, 550, 365

Frisch, M. J., Trucks, G. W., Schlegel, H. B., et al. 2009, Gaussian 09 (Wallingford, CT: Gaussian, Inc.)

Furuya, K., Aikawa, Y., Sakai, N., \& Yamamoto, S. 2011, ApJ, 731, 38

Giuliano, B. M., Bizzocchi, L., Pietropolli Charmet, A., et al. 2019, A\&A, 628, A53
Hollis, J. M., Lovas, F. J., \& Jewell, P. R. 2000, ApJL, 540, L107

Jones, P. A., Cunningham, M. R., Godfrey, P. D., \& Cragg, D. M. 2007, MNRAS, 374, 579

Kattija-Ari, M., \& Harmony, M. D. 1980, IJQC, 18, 443

Kaushik, V., \& Woods, R. 1982, ZPC, 132, 117

Kisiel, Z. 2015, PROSPE-Programs for Rotational Spectroscopy, http://info. ifpan.edu.pl/Bkisiel/orospe.htm

Kisiel, Z., Pszczółkowski, L., Medvedev, I. R., et al. 2005, JMoSp, 233, 231

Kraitchman, J. 1953, AmJPh, 21, 17

Kuan, Y., Charnley, S. B., Huang, H., Tseng, W., \& Kisiel, Z. 2003, ApJ, 593,848

Lesarri, A., Cocinero, E. J., López, J. C., \& Alonso, J. L. 2004, Angew. Chem Int. Ed., 43, 605

Loomis, R. A., Zaleski, D. P., Steber, A. L., et al. 2013, ApJL, 765, L9

Loru, D., Peña, I., Alonso, J. L., \& Eugenia Sanz, M. 2016, ChCom, 52, 3615

Mangum, J., \& Shirley, Y. 2015, PASP, 127, 266

Martin-Drumel, M.-A., Lee, K. L. K., Belloche, A., et al. 2019, A\&A, 623, A167

McGuire, B. A. 2018, ApJS, 239, 17

McGuire, B. A., Burkhardt, A. M., Kalenskii, S., et al. 2018, Sci, 359, 202

Müller, H. S., Schlöder, F., Stutzki, J., \& Winnewisser, G. 2005, JMoSt, 742,215

Neill, J. L., Harris, B. J., Steber, A. L., et al. 2013, OExpr, 21, 19743

Pearson, J. C., Sastry, K. V. L. N., Herbst, E., \& De Lucia, F. C. 1997, ApJ, 480, 420

Penn, R. E., \& Curl, R. F. 1971, JChPh, 55, 651

Pérez, C., Krin, A., Steber, A. L., et al. 2016, J. Phys. Chem. Lett., 7, 154

Pickett, H. M. 1991, JMoSp, 148, 371

Pickett, H. M., Poynter, R. L., Cohen, E. A., et al. 1998, JQSRT, 60, 883

Schmitz, D., Alvin Shubert, V., Betz, T., \& Schnell, M. 2012, JMoSp, 280, 77

Snyder, L. E., Lovas, F. J., Hollis, J. M., et al. 2005, ApJ, 619, 914

Western, C. M. 2017, JQSRT, 186, 221

Widicus, S., Drouin, B., Dyl, K., \& Blake, G. 2003, JMoSp, 217, 278

Zhang, D., Bocklitz, S., \& Zwier, T. S. 2016, JPCA, 120, 55

Zuckerman, B., Turner, B. E., Johnson, D. R., et al. 1975, ApJL, 196, L99 